TRANSACTIONS OF THE

AMERICAN MATHEMATICAL SOCIETY

Volume 351, Number 5, Pages 1765-1801

S 0002-9947(99)02103-0

Article electronically published on January 27, 1999

\title{
THE LIMIT SPACES OF TWO-DIMENSIONAL MANIFOLDS WITH UNIFORMLY BOUNDED INTEGRAL CURVATURE
}

\author{
TAKASHI SHIOYA
}

\begin{abstract}
We study the class of closed 2-dimensional Riemannian manifolds with uniformly bounded diameter and total absolute curvature. Our first theorem states that this class of manifolds is precompact with respect to the Gromov-Hausdorff distance. Our goal in this paper is to completely characterize the topological structure of all the limit spaces of the class of manifolds, which are, in general, not topological manifolds and even may not be locally 2 -connected. We also study the limit of 2-manifolds with $L^{p}$-curvature bound for $p \geq 1$.
\end{abstract}

\section{INTRODUCTION}

Let $\mathcal{M}(C, D)$ denote the set of isometry classes of closed 2-dimensional Riemannian manifolds $M$ with diameter $\leq D$ and total absolute curvature $c_{\text {abs }}(M):=$ $\int_{M}\left|K_{M}\right| \leq C$ for two constants $C, D>0$, where $K_{M}$ denotes the curvature function on $M$. Our first theorem is the following:

Theorem 1.1 (Precompactness Theorem). The class $\mathcal{M}(C, D)$ is precompact with respect to the Gromov-Hausdorff distance.

This theorem means that any sequence of manifolds in $\mathcal{M}(C, D)$ has a subsequence which converges to some compact metric space. The main purpose of this paper is to study the topological structure of the limit spaces. Before we describe our main results, let us see an example.

Example 1.1. Define a function $f:[0,2 / 3 \pi] \rightarrow \mathbf{R}$ by $f(0):=0$ and $f(x):=$ $x^{4}(1+\sin (1 / x))$ for $x \in(0,2 / 3 \pi]$ as shown in Figure 1 .

Then, we see that

$$
S:=\left\{(x, y, z) \in \mathbf{R}^{3} \mid y^{2}+z^{2}=f(x)^{2}, 0 \leq x \leq 2 / 3 \pi\right\}
$$

is a limit of $\mathcal{M}(C, D)$ for some $C, D>0$. In fact, $S$ is approximated by the smooth surface of revolution

$$
S_{\epsilon}:=\left\{(x, y, z) \in \mathbf{R}^{2} \mid y^{2}+z^{2}=(f(x)+\epsilon)^{2}, \epsilon \leq x \leq 2 / 3 \pi-\epsilon\right\} \text { for small } \epsilon>0,
$$

which is homeomorphic to a closed annulus. Attaching two suitable small caps to the two circle boundary components of $S_{\epsilon}$, we have a $C^{\infty}$-Riemannian manifold $M_{\epsilon}$ homeomorphic to a sphere such that $\mathcal{M}(C, D) \ni M_{\epsilon} \rightarrow S$ as $\epsilon \rightarrow 0$ for some

Received by the editors October 30, 1996 and, in revised form, March 26, 1997.

1991 Mathematics Subject Classification. Primary 53C20.

Key words and phrases. Total curvature, $L^{p}$-curvature bound, triangle comparison, the Gromov-Hausdorff convergence.

This work was partially supported by a Grant-in-Aid for Scientific Research from the Japan Ministry of Education, Science and Culture. 


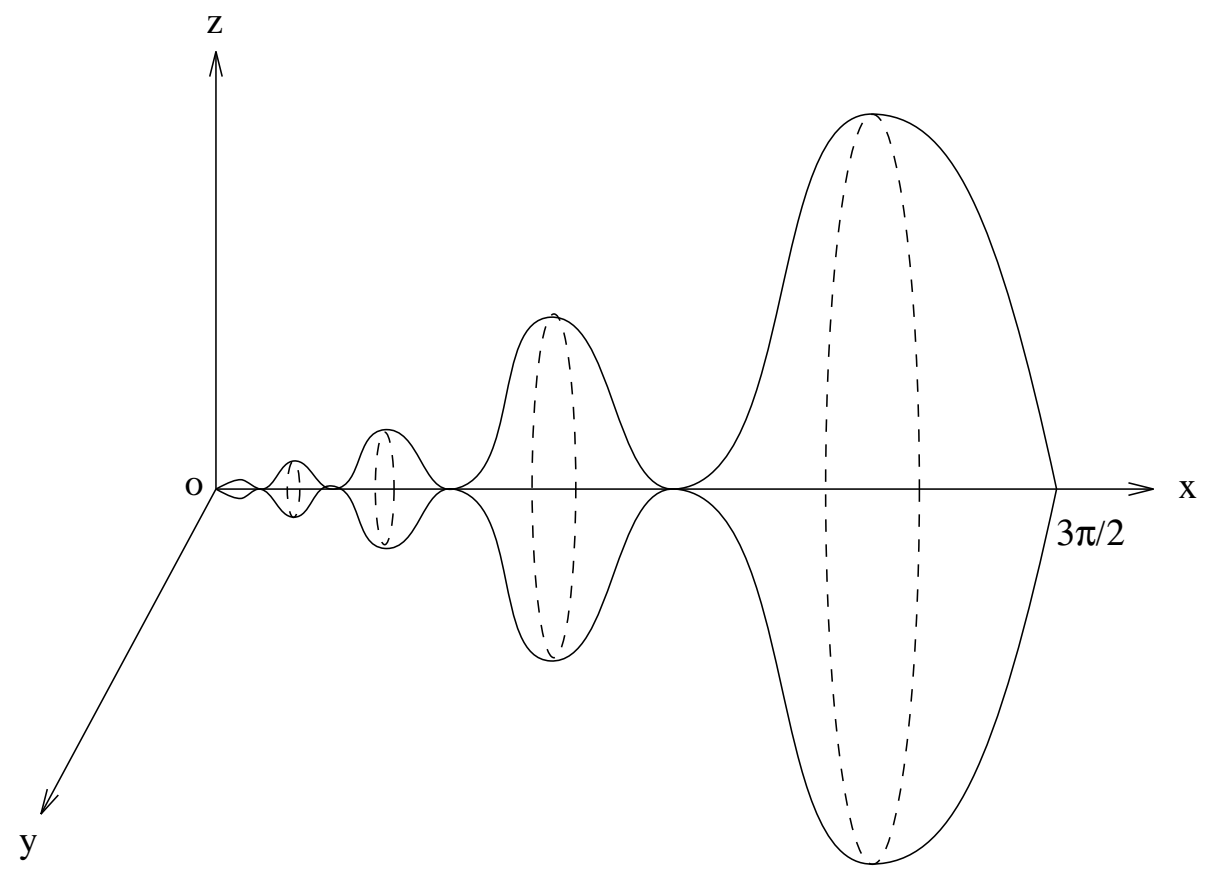

FiguRE 1

$C, D>0$, where the uniform boundedness of the total absolute curvature $c_{\text {abs }}\left(M_{\epsilon}\right)$ and the diameter of $M_{\epsilon}$ respectively follows from

$$
c_{\text {abs }}\left(S_{\epsilon}\right) \leq 2 \pi \int_{0}^{2 / 3 \pi}\left|f(t)^{\prime \prime}\right| d t<+\infty \quad \text { and } \quad \int_{0}^{2 / 3 \pi} \sqrt{1+f^{\prime}(t)^{2}} d t<+\infty .
$$

We observe that the 2-dimensional homotopy group $\pi_{2}(S)$ of $S$ is infinitely generated and $S$ is not locally 2 -connected.

In order to describe the topological structure of all the limit spaces of $\mathcal{M}(C, D)$, we give some definitions. Let $O$ be an open subset of the open line segment $L:=$ $\left\{(x, 0,0) \in \mathbf{R}^{3} \mid 0<x<1\right\}$. Then, $O$ is expressed as the countable union of open line segments $O_{i}=\left\{(x, 0,0) \mid x_{i}-\delta_{i}<x<x_{i}+\delta_{i}\right\}, i=1,2, \ldots$ Exclude each $O_{i}$ from $L$ and instead attach the two-point-punctured sphere $S\left(\left(x_{i}, 0,0\right), \delta_{i} ; \mathbf{R}^{3}\right)-$ $\left\{\left(x_{i}-\delta_{i}, 0,0\right),\left(x_{i}+\delta_{i}, 0,0\right)\right\}$ centered at $\left(x_{i}, 0,0\right)$ and of radius $\delta_{i}$ to obtain a subspace $S$ of $\mathbf{R}^{3}$, which we call the string of pearls associated with the open subset $O$. We call the closure $\bar{S}(=S \cup\{(0,0,0),(1,0,0)\})$ of $S$ a closed string of pearls, and the two points $(0,0,0)$ and $(1,0,0)$ the terminal points of the closed string of pearls $\bar{S}$. Notice that the space $S$ in Example 1.1 is homeomorphic to a closed string of pearls, and that an open punctured disk is homeomorphic to the string of pearls with $O=L$.

A topological space $X$ is called a pearl space if there exists an open neighborhood $U$ at each point $p \in X$ such that $U-\{p\}$ is homeomorphic to a finite disjoint union of strings of pearls. Here, we can always choose such a neighborhood $U$ satisfying that the closure $\bar{U}$ of $U$ is homeomorphic to the quotient space $\coprod_{i=1}^{k} \bar{S}_{i} /\left\{x_{1}, \ldots, x_{k}\right\}$ of the disjoint union $\coprod_{i=1}^{k} \bar{S}_{i}$ of finitely many closed strings of pearls $\bar{S}_{i}, i=1, \ldots, k$, 
where $x_{i} \in \bar{S}_{i}$ are terminal points. The number $k$ is called the index at $p \in X$ and denoted by $\operatorname{ind}(p)$. For instance, the following are all pearl spaces.

- (closed) strings of pearls,

- 2-dimensional topological manifolds,

- locally finite graphs (or 1-dimensional polytopes).

We have the following:

Theorem 1.2 (Topological Structure Theorem). Any limit space $X$ of the class $\mathcal{M}(C, D)$ is a compact pearl space and satisfies the following (1) and (2).

(1) We have

$$
\sum_{p \in X} \max \{\operatorname{ind}(p)-2,0\} \leq \frac{C}{2 \pi} .
$$

(2) The fundamental group $\pi_{1}(X)$ of $X$ is generated by at most $[2+C / 2 \pi]$ elements.

The converse of the above is true as described in the following:

Theorem 1.3. Any compact pearl space $X$ possesses a metric for which $X$ is a limit space of $\mathcal{M}(C, D)$ for some $C, D>0$.

We next consider the class $\mathcal{M}(p, C, D)$ of closed 2-dimensional Riemannian manifolds $M$ with $L^{p}$-norm of curvature $\int_{M}\left|K_{M}\right|^{p} \leq C$ and diameter $\leq D$ for given constants $p \geq 1, C, D>0$. It holds (see Proposition 8.1) that $\mathcal{M}(p, C, D) \subset \mathcal{M}\left(C^{\prime}, D\right)$, where $C^{\prime}$ is a constant depending only on $p, C$, and $D$, so that in particular, $\mathcal{M}(p, C, D)$ is also precompact.

Theorem 1.4. Let $X$ be a topological space and $p>1$ a number. Then, the following (1) and (2) are equivalent:

(1) There exists a metric on $X$ for which $X$ is a limit space of $\mathcal{M}(p, C, D)$ for some $C, D>0$.

(2) $X$ is a compact pearl space and satisfies that $\operatorname{ind}(x) \leq 2$ for any $x \in X$.

Our main theorems are connected to the earlier work due to Burago [5]. However, his paper [5] does not contain the details of the proofs, and our work is completely independent of his.

There are some other works on convergence of Riemannian manifolds under $L^{p_{-}}$ curvature bound and volume lower bound. See [23, 9] for the 2-dimensional case and $[24,15,16,17]$ for the higher dimensional case. However, since they all rely on some analytic methods to obtain the regularity of convergence of Riemannian metrics, it is impossible to extend them to the case where $p=1$ or to the case where the volume (even locally) tends to zero.

For the proof of our theorems (1.2 and 1.4), the triangle comparison theorem stated in the following plays an essential role. To state it we need some definitions. Over a given limit space $X$ of $\mathcal{M}(C, D)$ we find a (not necessarily unique) Radon measure $c_{\mathrm{abs}}^{X}$, the so-called absolute curvature measure, as the limit of some sequence of the absolute curvature measures $c_{\mathrm{abs}}^{M_{i}}:=c_{\mathrm{abs}}$ on $M_{i}$, where $M_{i}$, $i=1,2, \ldots$, are manifolds in $\mathcal{M}(C, D)$ converging to $X$ with respect to the GromovHausdorff distance (see for details $\S 4.3$ ). For any triangle $\triangle x_{1} x_{2} x_{3}$ in $X$, denote by $\tilde{\angle} x_{1} x_{2} x_{3}$ the angle at $\tilde{x}_{2}$ of a triangle $\triangle \tilde{x}_{1} \tilde{x}_{2} \tilde{x}_{3}$ in $\mathbf{R}^{2}$ such that $d\left(x_{i}, x_{j}\right)=d\left(\tilde{x}_{i}, \tilde{x}_{j}\right)$ for any $i, j=1,2,3$, where $d$ is the distance function. For $A, B \subset X$, let $K(A, B)$ 
be the set of points on all minimal segments joining $a \in A$ and $b \in B$. Then, we have:

Theorem 1.5 (Triangle Comparison Theorem). Let $\triangle p q r$ be a triangle in a limit space $X$ of $\mathcal{M}(C, D)$ and $s$ a point on a minimal segment joining $p$ and $q$. Then, there exist three minimal segments $\sigma$ from $p$ to $s, \tau$ from $s$ to $q$, and $\gamma$ from $s$ to $r$ such that

$$
\tilde{L} s p r \geq \tilde{L} q p r-\lambda \epsilon,
$$

where $\lambda>0$ is a calculable universal constant and

$$
\epsilon:=\min \left\{c_{\mathrm{abs}}^{X}(K(r, \sigma \cup \tau)-\{p, q\}), c_{\mathrm{abs}}^{X}(K(\{p, q\}, \gamma)-\{r\})\right\} .
$$

Note that, if $c_{\mathrm{abs}}^{X} \equiv 0$, Theorem 1.5 is equivalent to $X$ having nonnegative curvature in the sense of Alexandrov (see [6]).

Using the Triangle Comparison Theorem (1.5) enables us to define the angle between two minimal segments emanating from a common point in the limit space $X$ of $\mathcal{M}(C, D)$ and then to induce 'the space of directions', which is a generalized concept of the unit tangent sphere of Riemannian manifold. Toward proving the Topological Structure Theorem (1.2), we will develop geometry on the limit spaces $X$ of $\mathcal{M}(C, D)$. In order to analyze the topology of a small metric ball $B(p, R) \subset X$, $p \in X, R>0$, we need some alternative to the Morse theory for the distance function. It is however impossible to intactly generalize the Morse theory as done for Alexandrov spaces ([14]) because of the fact that a string of pearls may have infinitely many topological critical points. We get over this difficulty to consider the quotient metric space of $B(p, R)$ modulo the equivalence relation that two points be contained in a common connected component of some distance sphere $\partial B(p, \rho)$, $0<\rho<R$, which quotient space turns out to be isometric to a cone over a finite set. Seeing that each equivalent class is either a point or a circle, we thus prove that $X$ is a pearl space.

Remark 1.1.

(1) Without a bound of total absolute curvature, we can expect nothing about the limit spaces other than that they are intrinsic metric spaces. In fact, according to [8], for a given compact intrinsic metric space $X$ there exists a sequence of closed 2-manifolds converging to $X$. See $\S 2.5$ for the definition of intrinsic metric space.

(2) For $C, D>0$, let $\mathcal{M}_{-}(C, D)$ denote the class of closed 2-dimensional Riemannian manifolds $M$ with $\operatorname{diam} M \leq D$ and total negative curvature $c_{-}(M):=\int_{M} K_{-} \leq C$, where $K_{-}(p):=\max \left\{-K_{M}(p), 0\right\}$ for any $p \in M$. We have $\mathcal{M}_{-}(C, D) \subset \mathcal{M}(4 \pi+2 C, D)$ (see Lemma 2.1) and that all our theorems stated above (Theorems 1.1-1.5) are still true even if all $\mathcal{M}(C, D)$ 's are replaced with $\mathcal{M}_{-}(C, D)$ (see Theorem 6.3).

(3) For $p \geq 1, C, D>0$, let $\mathcal{M}_{-}(p, C, D)$ be the class of closed 2-dimensional Riemannian manifolds $M$ with $\operatorname{diam} M \leq D$ and $L^{p}$-norm of negative curvature function $\int_{M} K_{-}{ }^{p} \leq C$. Then, Theorem 1.4 is still true if $\mathcal{M}(p, C, D)$ is replaced with $\mathcal{M}_{-}(p, C, D)$ (see Theorem 8.1).

(4) Since all the discussions of the proofs of Theorems 1.1-1.5 are local, they also hold for any limit space of closed metric balls $\bar{B}\left(p_{i}, R ; M_{i}\right), i=1,2, \ldots$, with radius $R$ of (not necessarily closed) complete 2 -dimensional Riemannian 
manifolds $M_{i}$ such that $c_{\text {abs }}\left(B\left(p_{i}, 2 R ; M_{i}\right)\right) \leq C$ for any fixed constants $R, C>0$. By Proposition 3.1, this is true even if $c_{\mathrm{abs}}$ is replaced with $c_{-}$.

Acknowledgment. This work was done while the author visited the Department of Mathematics, University of Maryland, College Park. He would like to thank them for their hospitality during his stay there. He would also like to thank Professors K. Grove, L. Guijarro, Y. Machigashira, K. Sugahara, and T. Yamaguchi for valuable discussions and comments, and to thank the referee for carefully reading this paper and for helpful comments.

\section{Preliminaries}

2.1. Total curvature. Let $M$ be a 2-dimensional Riemannian manifold. The total positive curvature $c_{+}(D)$ of a locally Lebesgue measurable subset $D$ of $M$ is defined to be the (possibly improper) Lebesgue integral $\int_{D} K_{+}$over $D$ of the function $K_{+}$defined by $K_{+}(p):=\max \left\{K_{M}(p), 0\right\}$ for any $p \in M$, where $K_{M}$ is the curvature function of $M$. The total negative curvature $c_{-}(D)$ of $D$ is defined in the same manner for $K_{-}(p):=\max \left\{-K_{M}(p), 0\right\}$ instead of $K_{+}$. The total curvature $c(D)$ and the total absolute curvature $c_{\text {abs }}(D)$ of $D$ are respectively defined by $c(D):=c_{+}(D)-c_{-}(D)$ and $c_{\text {abs }}(D):=c_{+}(D)+c_{-}(D)$. Note that $c_{ \pm}(D)=+\infty$ may happen and we agree that $c(D)$ is defined only when one of $c_{-}(D)$ and $c_{+}(D)$ is finite. We sometimes write $c^{M}(D), c_{\mathrm{abs}}^{M}(D)$, etc. instead of $c(D), c_{\mathrm{abs}}(M)$, etc.

For a domain $D$ of $M$ bounded by piecewise smooth curves, we denote by $\angle_{p} D$ the inner angle at a point $p \in \partial D$, and by $\kappa(\partial D)$ the sum of exterior angles of $D$ and of the total geodesic curvature of $\partial D$ with respect to $D$, i.e., if $\partial D$ splits into $n$ unit-speed smooth curves $\alpha_{i}: \mathbf{R} \supset I_{i} \rightarrow \partial D, i=1, \ldots, n$ (where $n$ may be $\infty$ ) whose parameterizations are all positive with respect to $D$, then

$$
\kappa(\partial D):=\sum_{p \in \partial D}\left(\pi-\angle_{p} D\right)+\sum_{i=1}^{n} \int_{I_{i}} k_{\alpha_{i}}(s) d s
$$

where $k_{\alpha_{i}}$ is the geodesic curvature of $\alpha_{i}$. With these notations, the Gauss-Bonnet formula is described as

$$
c(D)+\kappa(\partial D)=2 \pi \chi(D)
$$

for any compact domain $D$ surrounded by finitely many piecewise smooth closed curves.

Lemma 2.1. For any closed 2-dimensional Riemannian manifold $M$, we have

$$
c_{\text {abs }}(M) \leq 4 \pi+2 c_{-}(M),
$$

and in particular,

$$
\mathcal{M}_{-}(C, D) \subset \mathcal{M}(4 \pi+2 C, D)
$$

Proof. It follows from the Gauss-Bonnet theorem that

$$
c_{+}(M)=2 \pi \chi(M)+c_{-}(M) \leq 4 \pi+c_{-}(M),
$$

which completes the proof. 
2.2. Variants of Landau's symbols. Denote by ' $\operatorname{const}_{\alpha, \beta, \ldots}$ ' the symbol expressing some constant depending only on $\alpha, \beta, \ldots$ In particular, 'const' means some universal constant. Let $O_{\alpha, \beta, \ldots}(\cdot), \omega_{\alpha, \beta, \ldots}(\cdot)$ be the symbols which express some functions depending only on $\alpha, \beta, \ldots$ such that $\lim \sup _{t \rightarrow 0}\left|O_{\alpha, \beta, \ldots}(t)\right| / t<+\infty$ and $\lim _{t \rightarrow 0} \omega_{\alpha, \beta, \ldots}(t)=0$ respectively. If the subscript is just ' $*$ ' such as const ${ }_{*}, O_{*}(\cdot)$, $\omega_{*}(\cdot)$, this means to depend possibly on everything.

2.3. Maximal net. Let $X$ be a metric space, $d$ the distance function on $X$, and $\epsilon$ a positive number. Denote by $\beta_{X}(\epsilon)$ the number of elements of a maximal net $N \subset X$ such that $d(p, q) \geq \epsilon$ for any different $p, q \in N$. Notice that $\beta_{X}(\epsilon)<+\infty$ for any $\epsilon>0$ if and only if $X$ is precompact. For $\alpha \geq 0$, denote by $\mathcal{H}^{\alpha}$ the $\alpha$ dimensional Hausdorff measure. Then, a straightforward discussion yields that for any $\alpha \geq 0$,

$$
\mathcal{H}^{\alpha}(X) \leq \text { const }_{\alpha} \cdot \limsup _{\epsilon \rightarrow 0} \epsilon^{-\alpha} \beta_{X}(\epsilon)
$$

2.4. (Measured) Gromov-Hausdorff topology. Let $Z$ be a metric space. The Hausdorff distance $d_{H}^{Z}(X, Y)$ between two compact subsets $X$ and $Y$ of $Z$ is defined by

$$
d_{H}^{Z}(X, Y):=\inf \{\rho>0 \mid B(X, \rho) \supset Y, B(Y, \rho) \supset X\},
$$

where $B(A, \rho):=B(A, \rho ; Z):=\{p \in Z \mid d(p, A)<\rho\}$ for $A \subset Z$ and $\rho>0$. Let $\mathcal{C}$ denote the set of isometry classes of compact metric spaces and $X, Y \in \mathcal{C}$. The Gromov-Hausdorff distance $d_{H}(X, Y)$ between $X$ and $Y$ is defined to be the infimum of $d_{H}^{Z}(f(X), g(Y))$, where $f: X \rightarrow Z$ and $g: Y \rightarrow Z$ are any isometric embeddings of $X, Y$ into any metric space $Z$. The $d_{H}$ is a distance function on $\mathcal{C}$ and determines a topology on $\mathcal{C}$, called the Gromov-Hausdorff topology. A $\delta$ approximation $\varphi: X \rightarrow Y, \delta>0$, is defined to satisfy

$$
\begin{gathered}
|d(\varphi(p), \varphi(q))-d(p, q)| \leq \delta \quad \text { for any } p, q \in X, \\
B(\varphi(X), \delta)=Y .
\end{gathered}
$$

Then, $d_{H}(X, Y) \leq O(\delta)$ if and only if there exists an $O(\delta)$-approximation from $X$ to $Y$.

Let $\mathcal{C} M$ denote the class of pairs $(X, \mu)$ of compact metric space $X$ and Borel measure $\mu$ over $X$ with $\mu(X) \leq 1$. Let $\left(X_{i}, \mu_{i}\right),(X, \mu) \in \mathcal{C} M, i=1,2, \ldots$ The sequence $\left\{\left(X_{i}, \mu_{i}\right)\right\}$ is said to converge to $(X, \mu)$ in the sense of the measured GromovHausdorff convergence if there exists a Borel measurable $\omega_{*}(1 / i)$-approximation $\varphi_{i}: X_{i} \rightarrow X$ for every $i$ such that

$$
\lim _{i \rightarrow \infty} \int_{X_{i}} f \circ \varphi_{i} d \mu_{i}=\int_{X} f d \mu
$$

for any continuous function $f$ on $X$. The topology of $\mathcal{C} M$ defined by the measured Gromov-Hausdorff convergence is called the measured Gromov-Hausdorff topology. Fukaya proved:

Proposition 2.1 (2.10 of [10]). The projection $\Pi: \mathcal{C} M \rightarrow \mathcal{C}$ is proper.

As a direct consequence of the proposition, we have:

Corollary 2.1. Assume that a sequence $\left\{X_{i}\right\}$ of compact metric spaces converges to a compact metric space $X$ in the sense of the Gromov-Hausdorff topology and $\mu_{i}$ is a Borel measure over each $X_{i}$ such that $\mu\left(X_{i}\right) \leq$ const. Then, there exists a 
Borel measure $\mu$ over $X$ such that some subsequence of $\left\{\left(X_{i}, \mu_{i}\right)\right\}$ tends to $(X, \mu)$ in the sense of the measured Gromov-Hausdorff topology.

Denote by $\mathcal{P} C$ the class of finitely compact pointed metric spaces $(X, p)$, where the finitely compactness of $X$ is defined by that the closed metric ball $\bar{B}(p, R):=$ $\{q \in X \mid d(p, q) \leq R\}$ for any $R>0$ is compact. Denote by $\mathcal{P} M C$ the class of pairs $(X, p, \mu)$, where $(X, p) \in \mathcal{P} C$ and $\mu$ is any Borel measure over $X$. A sequence $\left\{\left(X_{i}, p_{i}\right)\right\} \subset \mathcal{P} C$ (resp. $\left.\left\{\left(X_{i}, p_{i}, \mu_{i}\right)\right\} \subset \mathcal{P} M C\right)$ is said to converge to an $(X, p) \in \mathcal{P} C$ (resp. $(X, p, \mu) \in \mathcal{P} M C)$ in the sense of the pointed Gromov-Hausdorff convergence (resp. the measured and pointed Gromov-Hausdorff convergence) if for any fixed $R>0$ there exists an $\omega_{*}(1 / i)$-approximation $\varphi_{i}:\left(\bar{B}\left(p_{i}, R ; X_{i}\right), p_{i}\right) \rightarrow$ $(\bar{B}(p, R ; X), p)$ (resp. in addition to that, $\varphi_{i}$ is measurable and satisfies (2.3) for any continuous function $f$ on $\bar{B}(p, R ; X))$. This defines topologies on $\mathcal{P} C$ and $\mathcal{P} M C$ respectively.

2.5. Minimal segment and intrinsic metric space. Let $(X, d)$ be a metric space. The length $L(c)$ of a continuous curve $c:[a, b] \rightarrow X$ is defined by

$$
L(c):=\sup _{a=s_{0}<\cdots<s_{k}=b} \sum_{i=0}^{k-1} d\left(c\left(s_{i}\right), c\left(s_{i+1}\right)\right) .
$$

A continuous curve joining two points $p, q \in X$ is called a minimal segment if the curve attains the minimal length among all continuous curves joining $p$ and $q$. Of course, a minimal segment joining given two points does not necessarily exist and is not unique in general. We assume that all minimal segments are parametrized by arc-length. Denote by $p q$ one of minimal segments joining $p$ and $q$ (if any).

The intrinsic metric $\hat{d}$ induced from $d$ is defined by

$$
\hat{d}(p, q):=\inf _{c} L(c) \quad \text { for any } p, q \in X,
$$

where $c$ is any continuous curve joining $p$ and $q$. Here, we agree that if the two points $p$ and $q$ are contained in two different arcwise connected components, then $\hat{d}(p, q)=+\infty$. We say that $(X, d)$ is an intrinsic metric space if $d=\hat{d}$. When $X$ is a finitely compact intrinsic metric space, it follows that any two points in $X$ are joined by at least one minimal segment.

2.6. Triangle. Let $X$ be a metric space. For three points $p, q, r \in X$, we mean a triangle $\triangle p q r$ as consisting of three minimal segments $p q, q r$, and $r p$ in $X$. For a triangle $\triangle p q r$ in a Riemannian manifold, we indicate by $\angle p q r$ the angle between $p q$ and $q r$. A comparison triangle $\tilde{\triangle} p q r$ of a triangle $\triangle p q r$ in $X$ is defined to be a triangle in $\mathbf{R}^{2}$ whose edges have the same lengths as the corresponding ones of $\triangle p q r$, i.e., if we set $\triangle \tilde{p} \tilde{q} \tilde{r}:=\tilde{\triangle} p q r$, then $d(\tilde{p}, \tilde{q})=d(p, q), d(\tilde{q}, \tilde{r})=d(q, r)$, and $d(\tilde{r}, \tilde{p})=d(r, p)$. For a triangle $\triangle p q r$ in $X$, denote by $\tilde{\angle} p q r$ the angle of the comparison triangle $\tilde{\triangle} p q r$ at the vertex corresponding to $q$, i.e., if $\triangle \tilde{p} \tilde{q} \tilde{r}:=\tilde{\triangle} p q r$, then $\tilde{\angle} p q r:=\angle \tilde{p} \tilde{q} \tilde{r}$.

\section{Precompactness of $\mathcal{M}(C, D)$}

The purpose of this section is to prove the Precompactness Theorem (1.1). 
3.1. Volume estimate of metric balls. In this subsection, we will give the estimate of the volumes of metric balls by the total absolute curvature, which is needed for the proof of the Precompactness Theorem (1.1).

Assume that $M$ is a 2-dimensional complete Riemannian manifold and $p \in M$ a fixed point.

Definition 3.1 ([12]). A number $t>0$ is called an exceptional radius with respect to $p$ if there exists a cut point $q \in \partial B(p, t)$ to $p$ which satisfies at least one of the following three conditions:

(1) The point $q$ is a conjugate point to $p$ along some minimal geodesic segment joining $p$ and $q$.

(2) The two points $p$ and $q$ are connected by at least three different minimal segments.

(3) The two points $p$ and $q$ are connected by exactly two different minimal segments and the angle at $q$ between them is just equal to $\pi$.

Hartman ([12]) proved that the set of exceptional radii with respect to $p$ is of measure zero and the metric circle $\partial B(p, t)$ with any nonexceptional radius $t>0$ consists of finitely many piecewise smooth closed curves whose break points coincide with the cut points to $p$. Note that he indeed proved them only when $M$ is homeomorphic to $\mathbf{R}^{2}$; nevertheless this assumption is not essential in his proof and they extend to the case where $M$ is any 2-dimensional complete Riemannian manifold (cf. [20]).

The length $L(\partial B(p, \rho))$ of the metric sphere $\partial B(p, \rho)$ with any nonexceptional radius $\rho>0$ satisfies (see $[12,20]$ )

$$
L(\partial B(p, \rho)) \leq \int_{0}^{\rho} \kappa(\partial B(p, t)) d t,
$$

where we note that $\kappa(\partial B(p, t))$ is defined for all nonexceptional $t \in(0, \rho]$, i.e., for almost all $t \in(0, \rho]$. Since $L(\partial B(p, \rho))>0$ and since any exceptional radius is a limit of nonexceptional radii, we have

$$
\int_{0}^{\rho} \kappa(\partial B(p, t)) d t \geq 0 \quad \text { for any } \rho \geq 0 .
$$

Let $R>r>0$ be two fixed nonexceptional radii with respect to $p$. Denote by $\mathcal{S}$ the set of all connected components of $\partial B(p, r)$ homotopic to zero in $B(p, R)-$ $B(p, r)$. Any $S \in \mathcal{S}$ surrounds a disk domain, say $D_{S}$, in $B(p, R)-B(p, r)$. For any nonexceptional $t \in[r, R]$, set

$$
\chi(t):=\chi\left(\bar{B}(p, t) \cup \bigcup_{S \in \mathcal{S}} D_{S}\right) .
$$

Proposition 3.1. We have

$$
c_{+}(B(p, r))-2 \pi \chi(r) \leq \frac{2 \pi r+R c_{-}(B(p, R))}{R-r} .
$$

Proof. We first claim the following:

$$
\chi(\bar{B}(p, t)) \leq \chi(t) \leq \chi(r) \text { for any nonexceptional } t \in[r, R] .
$$

The first inequality is trivial. Let us prove the second. For a nonexceptional radius $t \in(r, R]$, let $C_{t, i}, i=1,2, \ldots$, be all the connected components of $\bar{B}(p, t)-B(p, r)$. 
Then, for any $j$ we have

$$
\chi\left(\bar{B}(p, r) \cup \bigcup_{i=1}^{j+1} C_{t, i}\right)-\chi\left(\bar{B}(p, r) \cup \bigcup_{i=1}^{j} C_{t, i}\right)=\chi\left(C_{t, j+1}\right),
$$

which is positive only if $C_{t, j+1}=D_{S}$ for $S:=\partial B(p, r) \cap C_{t, j+1} \in \mathcal{S}$. This proves (3.3).

Since $\kappa(\partial B(p, t))=2 \pi \chi(\bar{B}(p, t))-c(B(p, t))$ for all nonexceptional $t>0$, we have

$$
\begin{aligned}
0 & \leq \int_{0}^{R} \kappa(\partial B(p, t)) d t \\
& \leq \int_{0}^{r}\left(2 \pi+c_{-}(B(p, r))\right) d t+\int_{r}^{R}\left\{2 \pi \chi(r)+c_{-}(B(p, R))-c_{+}(B(p, r))\right\} d t,
\end{aligned}
$$

which completes the proof.

Proposition 3.2 (cf. [22]). Let $p \in M$ and $0<\rho<\operatorname{rad}(M, p):=\sup _{q \in M} d(p, q)$.

(1) If $\rho$ is nonexceptional with respect to $p$, then

$$
L(\partial B(p, \rho)) \leq\left(2 \pi+c_{-}(B(p, \rho))\right) \rho .
$$

(2) We have

$$
\operatorname{vol} B(p, \rho) \leq \frac{1}{2}\left(2 \pi+c_{-}(B(p, \rho))\right) \rho^{2},
$$

and in particular

$$
\operatorname{vol} M \leq \frac{1}{2}\left(2 \pi+c_{-}(M)\right)(\operatorname{diam} M)^{2} .
$$

(3) If $B(p, t)$ is homeomorphic to an open disk for any $t \in(0, \rho)$, then

$$
\operatorname{vol} B(p, \rho) \geq \frac{1}{2}\left(2 \pi-c_{+}(B(p, \rho))\right) \rho^{2} .
$$

Proof. (1): It follows from the Gauss-Bonnet formula that for any nonexceptional radius $t>0$,

$$
\kappa(\partial B(p, t))=2 \pi \chi(B(p, t))-c(B(p, t)),
$$

where one has $\chi(B(p, t)) \leq 1$ if $t<\operatorname{rad}(M, p)$, which together with (3.1) proves (1).

(2): Since

$$
\operatorname{vol} B(p, \rho)=\int_{0}^{\rho} L(\partial B(p, t)) d t
$$

(see [12]), (1) implies (2).

(3): Assume that $\rho$ satisfies the assumption of (3) and let $r \in(0, \rho)$ be any fixed nonexceptional radius. Denote by $\left\{q_{i}\right\}_{i=1, \ldots, m}$ the set of cut points to $p$ on $\partial B(p, r)$. There are exactly two minimal segments joining $p$ to each $q_{i}$, which surrounds a closed disk domain in $\bar{B}(p, r)$, say $D_{i}$. Let $F_{i}, i=1, \ldots, m$, be the connected components of $B(p, r)-\bigcup_{i} D_{i}$, each of which is an open disk domain bounded by a triangle. Since each $F_{i}$ contains no cut points, a straightforward calculation using the geodesic polar coordinate yields

$$
\operatorname{vol} F_{i}=\int_{0}^{r} \int_{0}^{t} \kappa\left(\partial B(p, s) \cap F_{i}\right) d s d t,
$$


where, by the Gauss-Bonnet formula, $\kappa\left(\partial B(p, s) \cap F_{i}\right)=\angle_{p} F_{i}-c\left(B(p, s) \cap F_{i}\right) \geq$ $\angle_{p} F-c_{+}\left(B(p, r) \cap F_{i}\right)$. Hence,

$$
\operatorname{vol} B(p, r) \geq \sum_{i=1}^{m} \operatorname{vol} F_{i} \geq \frac{1}{2}\left\{\left(\sum_{i} \angle_{p} F_{i}\right)-c_{+}\left(B(p, r) \cap \bigcup_{i} F_{i}\right)\right\} r^{2} .
$$

Applying the Gauss-Bonnet formula to each $D_{i}$ yields

$$
2 \pi-\sum_{i} \angle{ }_{p} F_{i}=\sum_{i} \angle{ }_{p} D_{i} \leq c_{+}\left(\bigcup_{i} D_{i}\right) .
$$

Therefore we have

$$
\operatorname{vol} B(p, r) \geq \frac{1}{2}\left(2 \pi-c_{+}(B(p, r))\right) r^{2} .
$$

By taking $r \rightarrow \rho$, this completes the proof.

3.2. Upper bound of the order of the maximal net. We first prove the following lemma.

Lemma 3.1. Let $B(p, \rho)$ be a metric ball in a complete Riemannian manifold. Then, if $B(p, \rho)$ is not simply connected, there exists a minimal geodesic loop in $B(p, \rho)$ with base point $p$ which is not homotopic to zero in $B(p, \rho)$.

Proof. Let $\alpha:[0, \ell] \rightarrow B(p, \rho)$ be any unit-speed smooth loop with base point $p$ which is not homotopic to zero in $B(p, \rho)$, and let $d:=\max _{s \in[0, \ell]} d(p, \alpha(s))$. There is a sequence $0=s_{0}<\cdots<s_{k}=\ell$ such that $s_{i+1}-s_{i} \leq \rho-d$ for any $i$. Denote by $\sigma_{i}$ a minimal segment from $p$ to $\alpha\left(s_{i}\right)$ for each $i$. Since $\alpha$ is not homotopic to zero, there is an $i_{0}$ such that $\hat{\alpha}:=\left.\sigma_{i_{0}} \cup \alpha\right|_{\left[s_{i_{0}}, s_{i_{0}}+1\right]} \cup \sigma_{i_{0}+1}$ is not homotopic to zero. It follows that

$$
L(\hat{\alpha})=d\left(p, \alpha\left(s_{i_{0}}\right)\right)+s_{i_{0}+1}-s_{i_{0}}+d\left(p, \alpha\left(s_{i_{0}+1}\right)\right) \leq d+\rho .
$$

Therefore, the minimal length of loops in $B(p, \rho)$ with base point $p$ which are not homotopic to zero is attained by a loop contained in $\bar{B}(p,(d+\rho) / 2) \subset B(p, \rho)$, which is a geodesic loop.

The following lemma is essential to prove the Precompactness Theorem (1.1).

Lemma 3.2. For any $M \in \mathcal{M}(C, D)$ and $\epsilon>0$, we have

$$
\beta_{M}(\epsilon) \leq \operatorname{const}_{C, D}\left(1+\epsilon^{-2}\right) .
$$

Proof. Let $M \in \mathcal{M}(C, D), \epsilon>0$, and let $N$ be a maximal net of $M$ such that $d(p, q) \geq \epsilon$ for any different $p, q \in N$, i.e., $\# N=\beta_{M}(\epsilon)$. To prove the lemma, it may be assumed that $\epsilon<\operatorname{diam} M$ and $\epsilon \ll 1$. Since all $B(p, \epsilon / 2), p \in N$, are disjoint to each other and by Proposition 3.2(2), setting $\delta:=\frac{\pi}{16} \epsilon^{2}$ one has

$$
\begin{aligned}
& \#\{p \in N \mid \operatorname{vol} B(p, \epsilon / 2) \geq \delta\} \leq \frac{\operatorname{vol} M}{\delta} \leq \frac{8}{\pi}(2 \pi+C) D^{2} \epsilon^{-2}, \\
& \#\left\{p \in N \mid c_{+}(B(p, \epsilon / 2)) \geq \delta\right\} \leq \frac{C}{\delta}=\frac{16}{\pi} C \epsilon^{-2} .
\end{aligned}
$$

Therefore, it suffices to estimate the number of elements of

$$
N^{\prime}:=\left\{p \in N \mid \operatorname{vol} B(p, \epsilon / 2)<\delta, c_{+}(B(p, \epsilon / 2))<\delta\right\} .
$$


Assume that $N^{\prime}$ contains at least two different points. For $p \in M$, we set

$\rho_{p}:=\sup \{\rho>0 \mid B(p, t)$ is homeomorphic to an open disk for any $t \in(0, \rho)\}$.

It clearly follows that $\rho_{p}>0$.

Sublemma 3.1. We have

$$
\rho_{p}<\epsilon / 2<\operatorname{rad}(M, p) \quad \text { for any } p \in N^{\prime} .
$$

Proof. By Proposition 3.2(3), one has for any $p \in N^{\prime}$ and for $t=\min \left\{\rho_{p}, \epsilon / 2\right\}$,

$$
\delta>\operatorname{vol} B(p, t) \geq\left(\pi-c_{+}(B(p, t))\right) t^{2}>(\pi-\delta) t^{2},
$$

and hence

$$
t<\sqrt{\frac{\delta}{\pi-\delta}}<\epsilon / 2
$$

which implies $t=\rho_{p}<\epsilon / 2$.

The assumption $\epsilon<\operatorname{diam} M$ proves $\epsilon / 2<\operatorname{rad}(M, p)$.

Next we prove:

Sublemma 3.2. The metric ball $B\left(p, \rho_{p}\right)$ for any $p \in N^{\prime}$ is homeomorphic to a disk.

Proof. Sublemma 3.1 shows that $\partial B\left(p, \rho_{p}\right) \neq \emptyset$. If $B\left(p, \rho_{p}\right)$ is not homeomorphic to a disk, there is a loop $\alpha$ with base point $p$ in $B\left(p, \rho_{p}\right)$ which is not homotopic to zero in $B\left(p, \rho_{p}\right)$. For any $\rho \in\left(0, \rho_{p}\right)$ close enough to $\rho_{p}$, the ball $B(p, \rho)$ contains $\alpha$, where $\alpha$ is still nonzero-homotopic because of $B(p, \rho) \subset B\left(p, \rho_{p}\right)$. This contradicts that $B(p, \rho)$ is homeomorphic to a disk.

Let $p \in N^{\prime}$ be fixed. There exists a sequence $\rho_{i} \searrow \rho_{p}$ such that $B\left(p, \rho_{i}\right)$ is not homeomorphic to a disk. By Lemma 3.1, we have a minimal geodesic loop $\gamma_{i}$ with base point $p$ in $B\left(p, \rho_{i}\right)$ which is not homotopic to zero in $B\left(p, \rho_{i}\right)$. Since $\left.\gamma_{i}\right|_{\left[0, L\left(\gamma_{i}\right) / 2\right]}$ is a minimal segment, one has $\rho_{i}>L\left(\gamma_{i}\right) / 2$. Now, if $L\left(\gamma_{i}\right) / 2<\rho_{p}$, then $\gamma_{i}$ is contained in $B\left(p, \rho_{p}\right)$, which contradicts that $B\left(p, \rho_{p}\right)$ is homeomorphic to a disk. Therefore we have $L\left(\gamma_{i}\right) / 2 \geq \rho_{p}$. There is a subsequence of $\left\{\gamma_{i}\right\}$ which converges to some simple geodesic loop $\gamma_{p}$ in $\bar{B}\left(p, \rho_{p}\right)$ such that $\left.\gamma_{p}\right|_{\left[0, L\left(\gamma_{p}\right) / 2\right]}$, $\left.\gamma_{p}\right|_{\left[L\left(\gamma_{p}\right) / 2, L\left(\gamma_{p}\right)\right]}$ are minimal segments and $L\left(\gamma_{p}\right) / 2=\rho_{p}$.

It follows from $\rho_{p}<\epsilon / 2$ (see Sublemma 3.1) that $\gamma_{p}$ and $\gamma_{q}$ do not intersect for any different $p, q \in N^{\prime}$. If a point $p \in N^{\prime}$ satisfies that $\gamma_{p}$ is homotopic to zero in $M$, then $\gamma_{p}$ bounds at least one disk domain in $M$. Denote by $\mathcal{D}$ the set of open disk domains bounded by all $\gamma_{p}, p \in N^{\prime}$, homotopic to zero, and by $\hat{\mathcal{D}}$ the set of minimal elements of $\mathcal{D}$ with respect to the inclusion relation. Obviously, $\hat{\mathcal{D}}$ is a family of disjoint disk domains in $M$ and hence $\hat{M}:=M-\bigcup \hat{\mathcal{D}}$ is a compact manifold possibly with boundary.

We observe that all the geodesic loops $\gamma_{p}, p \in N^{\prime}$, are no more homotopic to zero in $\hat{M}$. Since, by the Gauss-Bonnet formula, each disk domain in $\hat{\mathcal{D}}$ has total curvature $>\pi$, the number of elements of $\hat{\mathcal{D}}$ is $<C / \pi$. Since $|\chi(M)|=|c(M)| / 2 \pi \leq$ $C / 2 \pi$, one has $|\chi(\hat{M})|<3 C / 2 \pi$. Therefore, the number of free homotopy classes of $\hat{M}$ represented by simple closed curves is $\leq$ const $_{C}$. (See Figure 2.)

If two different geodesic loops $\gamma_{p}$ and $\gamma_{q}, p, q \in N^{\prime}$, are homotopic to each other in $\hat{M}$, then there is a domain $A \subset \hat{M}$ homeomorphic to an annulus such that 


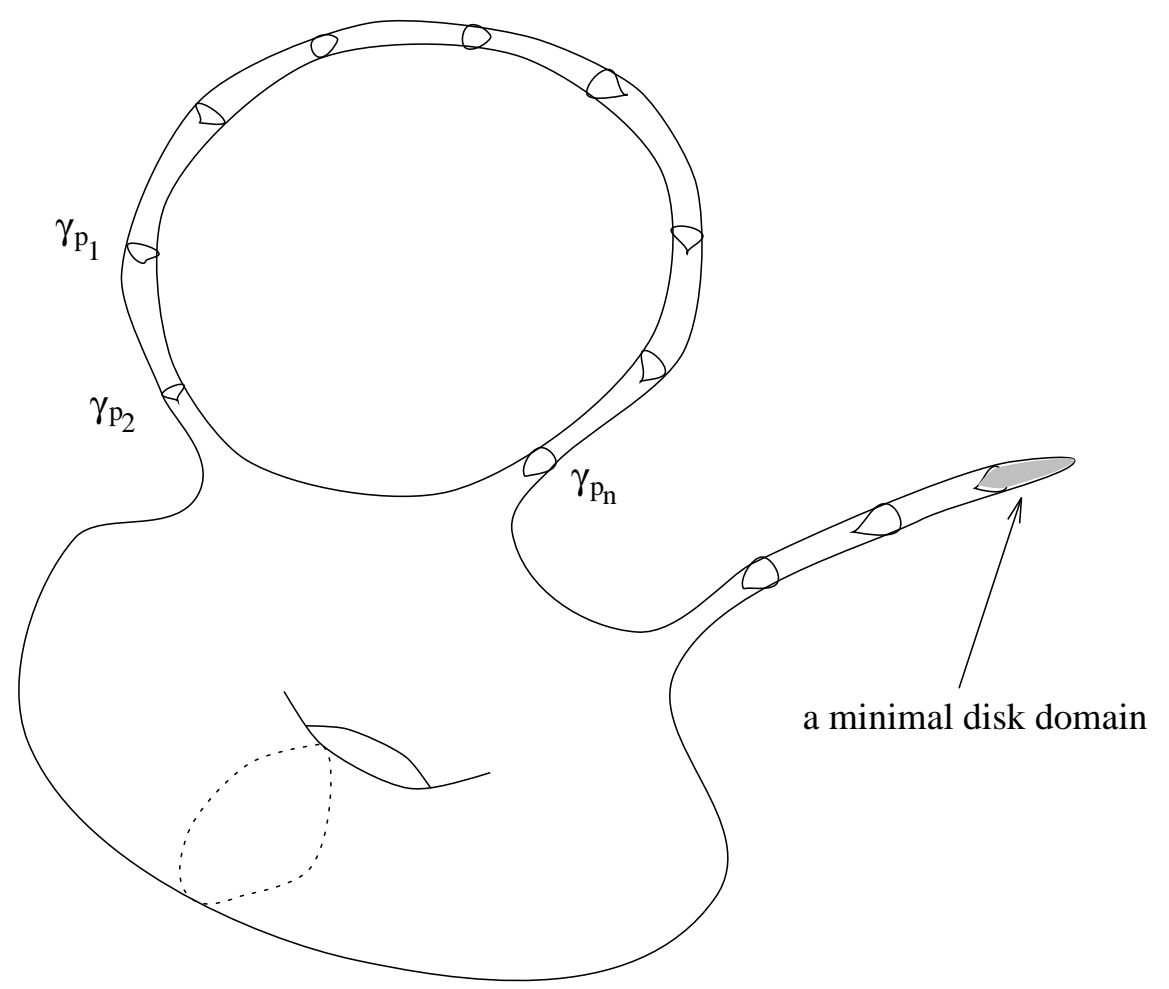

FiguRE 2

$\partial A=\gamma_{p} \cup \gamma_{q}$. Now, for a given nonzero free homotopy class $\mathcal{C} \in\left[S^{1} ; \hat{M}\right]$ represented by a simple closed curve, one finds all geodesic loops $\gamma_{p_{i}}$ with $p_{i} \in N^{\prime}$ belonging to the class $\mathcal{C}$. Assume that the number of $p_{i}$, say $n$, is not less than 2. Then, there are a domain $A_{\mathcal{C}}$ homeomorphic to an annulus and a permutation $\tau:\{1, \ldots, n\} \rightarrow$ $\{1, \ldots, n\}$ such that $\partial A_{\mathcal{C}}=\gamma_{p_{\tau(1)}} \cup \gamma_{p_{\tau(n)}}, \bigcup_{i=2, \ldots, n-1} \gamma_{p_{\tau(i)}}$ divides $A_{\mathcal{C}}$ into $n-1$ annuli, and $\gamma_{p_{\tau(1)}}, \ldots, \gamma_{p_{\tau(n)}}$ lie in $A_{\mathcal{C}}$ in this order. Since a minimal segment in $M$ joining $p_{\tau(1)}$ and $p_{\tau([n / 2])}$ has to intersect at least $[n / 2]-2$ geodesic loops $\gamma_{p_{\tau(i)}}$ with $i \neq 1,[n / 2]$ whose lengths are all less than $\epsilon / 2$, the triangle inequalities show that

$$
D \geq d\left(p_{\tau(1)}, p_{\tau([n / 2])}\right) \geq([n / 2]-1) \epsilon / 2,
$$

which implies $n \leq 4+4 D \epsilon^{-1}$. Recalling the number of all such classes $\mathcal{C}$ is $\leq$ const $_{C}$, we have

$$
\# N^{\prime} \leq\left(4+4 D \epsilon^{-1}\right) \operatorname{const}_{C} .
$$

This completes the proof of Lemma 3.2.

Proof of the Precompactness Theorem (1.1). The theorem follows from the same discussion as in the proof of the Gromov precompactness theorem ([11]) using Lemma 3.2.

Theorem 3.1. Any limit space $X \in \overline{\mathcal{M}(C, D)}$ satisfies

$$
\mathcal{H}^{2}(X) \leq \text { const }_{C, D}
$$


Proof. A straightforward discussion shows that Lemma 3.2 holds also for any limit $X \in \overline{\mathcal{M}(C, D)}$ instead of $M \in \mathcal{M}(C, D)$, which implies the theorem.

The same discussions using Proposition 3.1 prove the following lemma and theorem.

Lemma 3.3. If a complete pointed 2-dimensional Riemannian manifold $(M, p)$ satisfies $c_{\mathrm{abs}}(B(p, 2 R)) \leq C$ for given constants $C, R>0$, then

$$
\beta_{B(p, R)}(\epsilon) \leq \text { const }_{C, R}\left(1+\epsilon^{-2}\right) .
$$

Theorem 3.2. For any fixed $C, R>0$, any limit $(X, p)$ of complete pointed 2dimensional Riemannian manifolds $(M, q)$ such that $c_{\mathrm{abs}}(B(q, 2 R)) \leq C$ satisfies

$$
\mathcal{H}^{2}(B(p, R)) \leq \text { const }_{C, R} \text {. }
$$

\section{TRIANGLE COMPARISON}

In this section, we will prove the Triangle Comparison Theorem (1.5).

4.1. Almost flat triangular domains. A triangular domain $\mathbf{\Delta} p q r$ (or simply $\mathbf{\Lambda}$ ) is defined to be a 2-dimensional Riemannian manifold homeomorphic to a closed disk and surrounded by a triangle $\triangle p q r$ whose edges are all minimal geodesics. For $\epsilon>0$, we say that a triangular domain $\mathbf{\Delta}=\mathbf{\Delta} p q r$ is $\epsilon$-almost similar to a triangular domain $\boldsymbol{\Delta}^{\prime}=\boldsymbol{\Delta} p^{\prime} q^{\prime} r^{\prime}$ if

$$
\left|\angle_{p} \mathbf{\Lambda}-\angle_{p^{\prime}} \mathbf{\Lambda}^{\prime}\right| \leq \epsilon, \quad\left|\angle_{q} \mathbf{\Lambda}-\angle_{q^{\prime}} \mathbf{\Lambda}^{\prime}\right| \leq \epsilon, \quad\left|\angle_{r} \mathbf{\Lambda}-\angle_{r^{\prime}} \mathbf{\Lambda}^{\prime}\right| \leq \epsilon .
$$

The comparison triangular domain $\tilde{\mathbf{\Lambda}}=\tilde{\mathbf{\Lambda}} p q r$ of a triangular domain $\mathbf{\Lambda}=\mathbf{\Delta} p q r$ is defined to be the piece of $\mathbf{R}^{2}$ surrounded by the comparison triangle $\tilde{\triangle} p q r$. A triangular domain $\boldsymbol{\Lambda}=\boldsymbol{\Delta} p q r$ is said to be $\epsilon$-almost flat if $c_{\mathrm{abs}}(\mathbf{\Lambda}) \leq \epsilon$. It follows from the Gauss-Bonnet formula that any $\epsilon$-almost flat triangular domain $\boldsymbol{\Lambda}=\boldsymbol{\Lambda} p q r$ satisfies

$$
\left|\angle_{p} \mathbf{\Lambda}+\angle_{q} \mathbf{\Lambda}+\angle_{r} \mathbf{\Lambda}-\pi\right| \leq \epsilon .
$$

The following theorem is well known (see Lemma 6.3.1 of [19]).

Theorem 4.1. Any $\epsilon$-almost flat triangular domain $\mathbf{\Delta} p q r$ is $O(\epsilon)$-almost similar to its comparison triangular domain $\tilde{\mathbf{\Lambda}} p q r$.

4.2. Triangle comparison for manifolds. Throughout this subsection, let $M$ be a complete 2-dimensional Riemannian manifold.

It is easy to prove the following:

Lemma 4.1. If two triangles $\triangle p q r$ and $\triangle p^{\prime} q^{\prime} r^{\prime}$ in $\mathbf{R}^{2}$ satisfy

$$
1 \leq \frac{d(p, q)}{d\left(p^{\prime}, q^{\prime}\right)} \leq 1+\epsilon, \quad d(p, r)=d\left(p^{\prime}, r^{\prime}\right), \quad \text { and } \quad d(q, r)=d\left(q^{\prime}, r^{\prime}\right),
$$

then

$$
\angle p q r \leq \angle p^{\prime} q^{\prime} r^{\prime}+O\left(\epsilon^{1 / 2}\right) .
$$

Lemma 4.2. Any triangle $\triangle p q r$ in $M$ (which does not necessarily bound a triangular domain) satisfies

$$
\angle p q r \geq \tilde{\angle} p q r-O\left(c_{\mathrm{abs}}(K(r, p q))\right),
$$

where $K(\cdot, \cdot)$ is as in the Triangle Comparison Theorem (1.5). 
Proof. If the triangle $\triangle p q r$ degenerates, i.e., the sum of the lengths of two edges is equal to the length of the other, then the lemma is trivial. Assume thus that $\triangle p q r$ is nondegenerate. Set $\gamma:=p q$ and for each $t \in[0, d(p, q)]$ denote by $\mathcal{S}_{t}^{-}$ (resp. $\mathcal{S}_{t}^{+}$) the set of minimal segments $\sigma$ from $\gamma(t)$ to $r$ such that $\angle(\dot{\sigma}(0),-\dot{\gamma}(t))$ (resp. $\angle(\dot{\sigma}(0), \dot{\gamma}(t)))$ attains its minimum among all choices of minimal segments from $\gamma(t)$ to $r$. Note here that there are at most two element of $\mathcal{S}_{t}^{-}$(resp. $\mathcal{S}_{t}^{+}$) for each $t$, because $M$ is 2-dimensional. Define

$$
\begin{aligned}
\mathcal{T}:=\{\boldsymbol{\Delta} \subset M \mid \boldsymbol{\Delta} & \text { is the triangular domain surrounded by } \sigma, \tau, \text { and }\left.\gamma\right|_{[s, t]} \text { for } \\
& \text { some } \left.s, t \in[0, d(p, q)] \text { with } s<t \text { and for some } \sigma \in \mathcal{S}_{s}^{+}, \tau \in \mathcal{S}_{t}^{-}\right\} .
\end{aligned}
$$

For $\boldsymbol{\Delta} \in \mathcal{T}$, let $s(\mathbf{\Lambda}), t(\mathbf{\Delta}), \sigma_{\mathbf{\Lambda}}, \tau_{\mathbf{\Lambda}}$ denote the above $s, t, \sigma, \tau$ associated with $\boldsymbol{\Lambda}$. For $\delta \in(0, \operatorname{conv}(K(r, p q)) / 4)$, where $\operatorname{conv}(\cdot)$ denotes the convexity radius, we put

$$
\mathcal{T}_{\delta}:=\{\boldsymbol{\Delta} \in \mathcal{T} \mid \boldsymbol{\Delta} \subset \bar{B}(K(r, p q), \delta)\} .
$$

Let us now prove the following:

Sublemma 4.1. For any $s \in[0, d(p, q))$ there exists a triangular domain $\boldsymbol{\Lambda} \in \mathcal{T}_{\delta}$ such that $s(\mathbf{\Lambda})=s$.

Proof. For a given $s \in[0, d(p, q))$ one finds a sequence $s_{i} \searrow s, i=1,2, \ldots$, and minimal segments $\sigma_{i} \in \mathcal{S}_{s_{i}}^{-}$. There is a subsequence $\left\{\sigma_{i(j)}\right\}$ of $\left\{\sigma_{i}\right\}$ converging to a minimal segment $\sigma$ from $\gamma(s)$ to $r$. A standard discussion using the first variation formula shows that $\sigma$ is an element of $\mathcal{S}_{s}^{+}$. For each sufficiently large $j$, the segment $\sigma_{i(j)}$ is contained in $\bar{B}(\sigma, \delta)$, and hence $\sigma, \sigma_{i(j)}$, and $\left.\gamma\right|_{\left[s, s_{i(j)}\right]}$ together bound a triangular domain contained in $\bar{B}(\sigma, \delta)$, which is an element of $\mathcal{T}_{\delta}$.

Since any limit of triangular domains in $\mathcal{T}_{\delta}$ is also a triangular domain in $\mathcal{T}_{\delta}$, the above sublemma implies that for any $s \in[0, d(p, q))$ there exists a triangular domain $\mathbf{\Delta}_{\delta, s} \in \mathcal{T}_{\delta}$ with $s\left(\boldsymbol{\Lambda}_{\delta, s}\right)=s$ such that

$$
t\left(\mathbf{\Lambda}_{\delta, s}\right)=\max \left\{t(\mathbf{\Lambda}) \mid \mathbf{\Lambda} \in \mathcal{T}_{\delta}, s(\mathbf{\Lambda})=s\right\} .
$$

Now, we define a finite or infinite monotone increasing sequence of numbers $t_{0}, t_{1}, \ldots$ recursively by $t_{0}:=0$ and $t_{i+1}:=t\left(\boldsymbol{\Lambda}_{\delta, t_{i}}\right)$ whenever $t_{i}<d(p, q)$. For simplicity, set $\boldsymbol{\Delta}_{i}:=\boldsymbol{\Delta}_{\delta, t_{i}}, \sigma_{i}:=\sigma_{\mathbf{\Delta}_{i}}$ and $\tau_{i}:=\tau_{\mathbf{\Delta}_{i}}$. We will prove that $t_{n}=$ $d(p, q)$ for some $n$. Suppose the contrary, so that $\left\{t_{i}\right\}$ is an infinite monotone increasing sequence tending to some number $t_{\infty} \in(0, d(p, q)]$. By replacing with a subsequence, the segment $\sigma_{i}$ tends to some minimal segment $\sigma_{\infty}$ from $\gamma\left(t_{\infty}\right)$ to $r$. For a sufficiently large $i$, the segments $\sigma_{i}, \sigma_{\infty}$, and $\left.\gamma\right|_{\left[t_{i}, t_{\infty}\right]}$ together bound a triangular domain contained in $B\left(\sigma_{\infty}, \delta\right)$, which is an element of $\mathcal{T}_{\delta}$ (cf. the discussion in the proof of Sublemma 4.1). This contradicts the maximality of $t\left(\boldsymbol{\Lambda}_{i}\right)$, so that there is an $n$ such that $t_{n}=d(p, q)$. (See Figure 3.)

Let $\epsilon_{i}:=c_{\mathrm{abs}}\left(\boldsymbol{\Lambda}_{i}\right)$. Then, Theorem 4.1 implies that for each $i=0, \ldots, n-$ 1 the triangular domain $\boldsymbol{\Lambda}_{i}$ is $O\left(\epsilon_{i}\right)$-almost similar to its comparison triangular domain $\tilde{\mathbf{\Lambda}}_{i}$. Identify the edge of $\tilde{\mathbf{\Lambda}}_{i-1}$ corresponding to $\tau_{i-1}$ with the edge of $\tilde{\mathbf{\Lambda}}_{i}$ corresponding to $\sigma_{i}$ for every $i$. Then, the union $\tilde{D}_{\delta}:=\bigcup_{i=0}^{n} \tilde{\mathbf{\Lambda}}_{i}$ is bounded by the two line segments corresponding to $p r, q r$, say $\tilde{p} \tilde{r}, \tilde{q} \tilde{r}$, and the broken line segment corresponding to $\gamma$, say $\tilde{\gamma}$. Since $L(\tilde{\gamma})=d(p, q)<d(\tilde{p}, \tilde{r})+d(\tilde{q}, \tilde{r})$, one has $\angle_{\tilde{r}} \tilde{D}_{\delta}=\sum_{i=0}^{n-1} \angle_{\tilde{r}} \mathbf{\Lambda}_{i}<\pi$, so that $\tilde{D}_{\delta}$ may be assumed to be isometrically embedded 

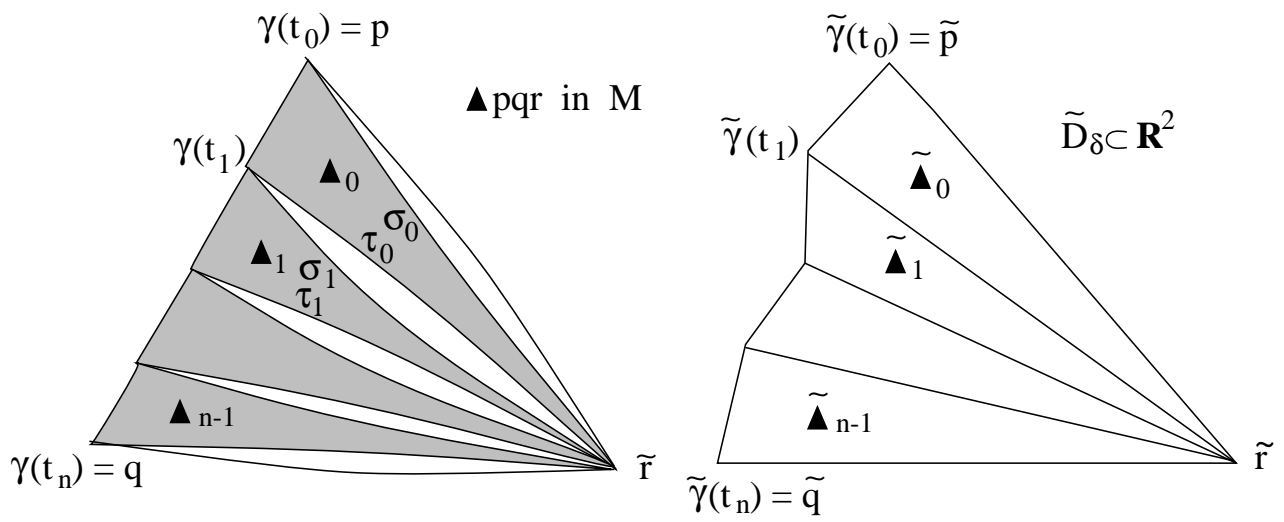

Figure 3

into $\mathbf{R}^{2}$. For any $i=1, \ldots, n-1$,

$$
\begin{aligned}
\angle_{\tilde{\gamma}\left(t_{i}\right)} \tilde{D}_{\delta} & =\angle_{\tilde{\gamma}\left(t_{i}\right)} \tilde{\mathbf{\Lambda}}_{i-1}+\angle_{\tilde{\gamma}\left(t_{i}\right)} \tilde{\mathbf{\Lambda}}_{i}=\angle_{\gamma\left(t_{i}\right)} \mathbf{\Lambda}_{i-1}+\angle_{\gamma\left(t_{i}\right)} \mathbf{\Lambda}_{i}+O\left(\epsilon_{i-1}+\epsilon\right) \\
& \leq \pi+O\left(\epsilon_{i-1}+\epsilon_{i}\right),
\end{aligned}
$$

where the last inequality follows from $\tau_{i-1} \in \mathcal{S}_{t_{i-1}}^{-}$and $\sigma_{i} \in \mathcal{S}_{t_{i}}^{+}$. Since $\mathbf{\Delta}_{i}^{\circ} \cap \mathbf{\Delta}_{j}^{\circ}=\emptyset$ for any $i \neq j$ (where $A^{\circ}$ is the interior of $A$ ), which we will prove later (see Sublemma $4.2)$, one has

$$
\sum_{i=0}^{n-1} \epsilon_{i} \leq c_{\mathrm{abs}}(B(K(r, p q), \delta))=: \epsilon(\delta) .
$$

Thus,

$$
\kappa_{-}(\tilde{\gamma}):=-\sum_{i=1}^{n-1} \min \left\{\pi-\angle_{\tilde{\gamma}\left(t_{i}\right)} \tilde{D}_{\delta}, 0\right\} \leq O(\epsilon(\delta)) .
$$

Let $k \in\{1, \ldots, n-1\}$ be a number such that $\tilde{\gamma}\left(t_{k}\right)$ is a convex vertex of $\tilde{D}_{\delta}$. We stretch the hinge $\left.\tilde{\gamma}\right|_{\left[t_{k-1}, t_{k+1}\right]}$ keeping $d\left(p, \tilde{\gamma}\left(t_{i}\right)\right)$ for all $i \neq k$ and $d\left(\tilde{\gamma}\left(t_{i}\right), \tilde{\gamma}\left(t_{i+1}\right)\right)$ for all $i$ to obtain a new broken line segment $\tilde{\gamma}^{\prime}$ such that

$$
\begin{aligned}
& L\left(\tilde{\gamma}^{\prime}\right)=L(\tilde{\gamma}), \quad \angle_{\tilde{\gamma}^{\prime}\left(t_{k-1}\right)} \tilde{D}_{\delta}^{\prime} \leq \angle_{\tilde{\gamma}\left(t_{k-1}\right)} \tilde{D}_{\delta}, \\
& \angle_{\tilde{\gamma}^{\prime}\left(t_{k}\right)} \tilde{D}_{\delta}^{\prime}=\pi, \quad \angle_{\tilde{\gamma}^{\prime}\left(t_{k+1}\right)} \tilde{D}_{\delta}^{\prime} \leq \angle_{\tilde{\gamma}\left(t_{k+1}\right)} \tilde{D}_{\delta},
\end{aligned}
$$

where $D_{\delta}^{\prime}$ is the domain surrounded by $\tilde{\gamma}^{\prime}, \tilde{\gamma}^{\prime}(0) \tilde{r}$, and $\tilde{\gamma}^{\prime}\left(t_{n}\right) \tilde{r}$. In particular, one has $\kappa_{-}\left(\tilde{\gamma}^{\prime}\right) \leq \kappa_{-}(\tilde{\gamma})$. Repeating such a stretching until there are no more convex vertices on the broken line segment, we eventually obtain a concave broken line segment $\tilde{\gamma}^{*}$ joining a point $\tilde{p}^{*}$ to a point $\tilde{q}^{*}$ which satisfies

$$
\begin{gathered}
d\left(\tilde{p}^{*}, \tilde{r}\right)=d(\tilde{p}, \tilde{r})=d(p, r), \quad d\left(\tilde{q}^{*}, \tilde{r}\right)=d(\tilde{q}, \tilde{r})=d(q, r), \quad L\left(\tilde{\gamma}^{*}\right)=L(\tilde{\gamma})=d(p, q), \\
\kappa_{-}\left(\tilde{\gamma}^{*}\right) \leq \kappa_{-}(\tilde{\gamma}), \quad \angle_{\tilde{p}^{*}} \tilde{D}_{\delta}^{*} \leq \angle_{\tilde{p}} \tilde{D}_{\delta}, \quad \angle_{\tilde{q}^{*}} \tilde{D}_{\delta}^{*} \leq \angle_{\tilde{q}} \tilde{D}_{\delta},
\end{gathered}
$$

where $\tilde{D}_{\delta}^{*}$ denotes the domain surrounded by $\tilde{\gamma}^{*} \cup \tilde{p}^{*} r \cup \tilde{q}^{*} r$. It follows from $\kappa_{-}\left(\tilde{\gamma}^{*}\right) \leq$ $O(\epsilon(\delta))$ that $\angle\left(v_{\tilde{\gamma}^{*}(t) \tilde{p}^{*}}, \tilde{\gamma}^{*}(t-0)\right) \leq O(\epsilon(\delta))$ for any $t \in(0, d(p, q)]$, where $v_{x y}$ for $x, y \in \mathbf{R}^{2}$ is the unit vector in $T_{x} \mathbf{R}^{2}$ identified with $(y-x) /|y-x|$. Hence, 
the first variation formula to the variation consisting of the line segments $\tilde{p}^{*} \tilde{\gamma}^{*}(t)$, $t \in[0, d(p, q)]$, shows

$$
1 \leq \frac{d(p, q)}{d\left(\tilde{p}^{*}, \tilde{q}^{*}\right)} \leq 1+O\left(\epsilon(\delta)^{2}\right) .
$$

Applying Lemma 4.1 to $\widetilde{\triangle p q r}$ and $\triangle \tilde{p}^{*} \tilde{q}^{*} r$ yields $\tilde{\angle} p q r \leq \angle \tilde{p}^{*} \tilde{q}^{*} r+O(\epsilon(\delta))$, and therefore,

$$
\begin{aligned}
\tilde{L} p q r & \leq \angle \tilde{p}^{*} \tilde{q}^{*} \tilde{r}+O(\epsilon(\delta))=\angle \tilde{q}^{*} \tilde{D}_{\delta}^{*}+O(\epsilon(\delta)) \leq \angle_{\tilde{q}} \tilde{D}_{\delta}+O(\epsilon(\delta)) \\
& =\angle{ }_{q} \mathbf{\Delta}_{n-1}+O(\epsilon(\delta)) \leq \angle p q r+O(\epsilon(\delta)) .
\end{aligned}
$$

Since $\epsilon(\delta)$ tends to $c_{\text {abs }}(K(r, p q))$ as $\delta \rightarrow 0$, this proves Lemma 4.2.

Sublemma 4.2. We have $\boldsymbol{\Lambda}_{i}^{\circ} \cap \mathbf{\Lambda}_{j}^{\circ}=\emptyset$ for any $i \neq j$.

Proof. Without loss of generality, it may be assumed that $i<j$. The minimal property of the segments $\gamma, \sigma_{i}, \tau_{i}, \sigma_{j}$, and $\tau_{j}$ shows that

$$
\partial \mathbf{\Delta}_{i} \cap \partial \mathbf{\Delta}_{j}= \begin{cases}\{r\} & \text { if } i+1<j, \\ \left\{r, \gamma\left(t_{j}\right)\right\} & \text { if } i+1=j \text { and } \tau_{i} \neq \sigma_{j}, \\ \tau_{i} & \text { if } i+1=j \text { and } \tau_{i}=\sigma_{j} .\end{cases}
$$

If $\left.\mathbf{\Lambda}_{i} \supset \gamma\right|_{\left[t_{i+1}, d(p, q)\right]}$, then $\left.\gamma\right|_{\left[t_{i+1}, t_{j+1}\right]} \cup \tau_{j}$ divides $\mathbf{\Delta}_{i}$ into two triangular domains as shown in Figure 4, the one of which surrounded by $\left.\gamma\right|_{\left[t_{i}, t_{j+1}\right]} \cup \sigma_{i} \cup \tau_{j}$ is an element of $\mathcal{T}_{\delta}$. Since $i<j$, this contradicts the maximality of $t\left(\boldsymbol{\Lambda}_{i}\right)$, so that we have $\left.\mathbf{\Lambda}_{i} \cap \gamma\right|_{\left(t_{i+1}, d(p, q)\right]}=\emptyset$. The same discussion yields $\left.\boldsymbol{\Delta}_{j} \cap \gamma\right|_{\left[0, t_{j}\right)}=\emptyset$. Therefore, if $i+1<j$, we have $\boldsymbol{\Lambda}_{i} \cap \mathbf{\Lambda}_{j}=\{r\}$, which implies the sublemma. If one supposes $i+1=j$ and $\boldsymbol{\Delta}_{i}^{\circ} \cap \boldsymbol{\Delta}_{j}^{\circ} \neq \emptyset$, then both $\boldsymbol{\Delta}_{i}$ and $\boldsymbol{\Delta}_{j}$ must contain the disk domain bounded by the biangle $\sigma_{j} \cup \tau_{i}$. This means that $\angle\left(\dot{\sigma}_{j}(0), \dot{\gamma}\left(t_{j}\right)\right)<\angle\left(\dot{\tau}_{i}(0), \dot{\gamma}\left(t_{j}\right)\right)$, which contradicts $\sigma_{j} \in S_{t_{i}}^{+}$and $\tau_{i} \in S_{t_{j}}^{-}$.

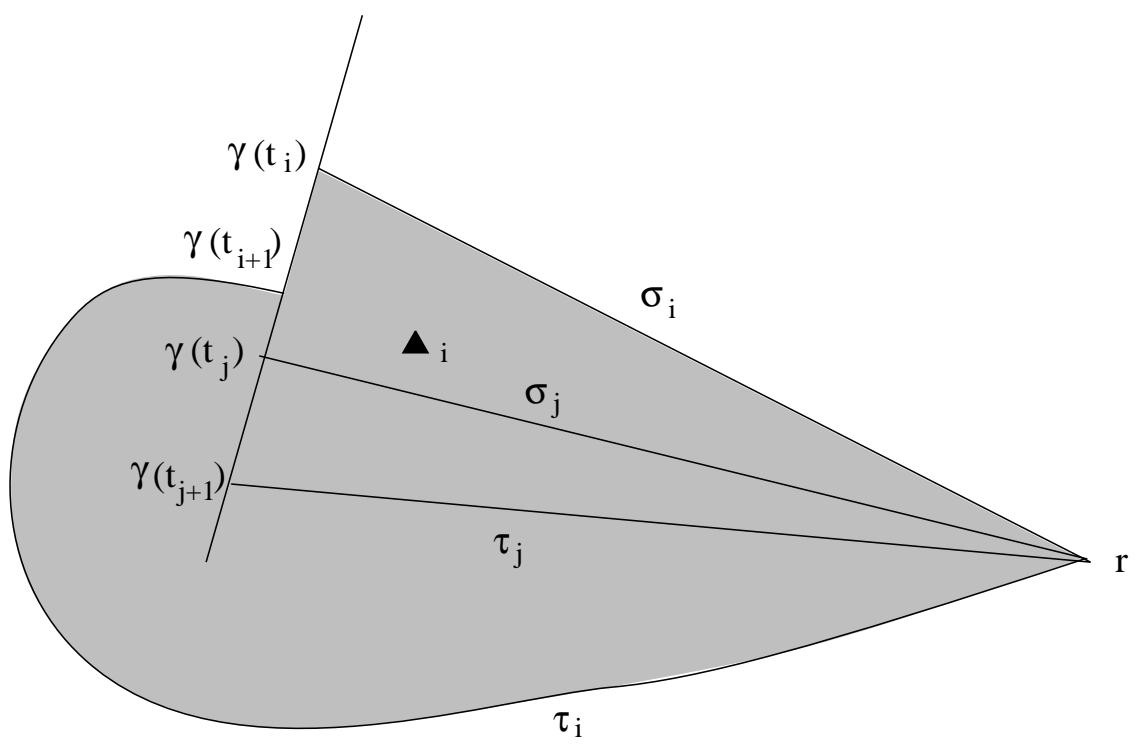

Figure 4 
For any triangle $\triangle p q r$ in a metric space, we define

$$
e(\triangle p q r):=\frac{d(p, q)+d(p, r)}{d(q, r)}-1 .
$$

Clearly, this is always nonnegative. It follows from a direct calculation that

$$
\begin{gathered}
\tilde{\angle} p q r=O\left(\sqrt{\frac{d(p, r)}{d(p, q)} e(\triangle p q r)}\right), \\
e(\triangle p q r)=O\left((\pi-\tilde{\angle} q p r)^{2}\right) .
\end{gathered}
$$

Lemma 4.3. Let $\triangle p q r$ be a triangle in a metric space, $s \in p q$ a point, and $\epsilon>0$ a number. Then, if $\tilde{\angle} p s r+\tilde{\angle} q s r \leq \pi+\epsilon$, we have

$$
\tilde{\angle} s q r \geq \tilde{\angle} p q r-O(\epsilon) \text {. }
$$

Proof. We glue the two comparison triangle domains $\tilde{\mathbf{\Lambda}} p s r=: \mathbf{\Delta} \tilde{p} \tilde{s} \tilde{r}$ and $\tilde{\mathbf{\Lambda}} s q r=$ : $\mathbf{\Delta} \tilde{s} \tilde{q} \tilde{r}$ at the edge $\tilde{s} \tilde{r}$ to obtain a quadrangle $\tilde{p} \tilde{s} \tilde{q} \tilde{r}$, which we embed into $\mathbf{R}^{2}$.

If $\tilde{s}$ is a convex vertex of the quadrangle, or $\tilde{\angle} p s r+\tilde{L} q s r \leq \pi$, then one obviously has $\tilde{L} s q r \geq \tilde{\angle} p q r$, which implies the lemma.

Assume that $\tilde{s}$ is a concave vertex, i.e., $\tilde{L} p s r+\tilde{\angle} q s r \geq \pi$. Since $\angle \tilde{p} \tilde{s} \tilde{q} \geq \pi-\epsilon$, applying (4.2) for $\triangle \tilde{p} \tilde{s} \tilde{q}$ yields

$$
1 \leq \frac{d(p, q)}{d(\tilde{p}, \tilde{q})}=1+O\left(\epsilon^{2}\right) .
$$

Hence, applying Lemma 4.1 completes the proof.

Lemma 4.4. For any triangle $\triangle p q r$ in $M$ and for any number $\rho$ with $0<\rho \ll 1$, we have

$$
\angle p q r \geq \tilde{\angle} p q r-O\left(\epsilon_{\rho}\right)-O_{\mu}\left(\rho^{1 / 4}\right)
$$

where

$$
\begin{aligned}
\gamma & :=p q, \\
t_{\rho} & :=\sup \{t \in[0, d(p, q)] \mid \gamma(t) r \cap B(p, \rho) \neq \emptyset \text { for some minimal } \\
& \text { segment } \gamma(t) r \text { joining } \gamma(t) \text { and } r\} \\
\epsilon_{\rho} & :=c_{\mathrm{abs}}\left(K\left(r,\left.\gamma\right|_{\left(t_{\rho}, d(p, q)\right]}\right)\right) \\
\mu & :=(d(p, q), d(q, r), d(r, p)),
\end{aligned}
$$

and where $O_{\mu}(\cdot)$ is continuous in $\mu$.

Proof. If $t_{\rho}<\rho^{1 / 2}$, applying Lemma 4.2 to $\triangle x q r, x:=\gamma\left(\rho^{1 / 2}\right)$, yields

$$
\angle p q r=\angle x q r \geq \tilde{\angle} x q r-O\left(\epsilon_{\rho}\right) .
$$

By $d(p, x)=\rho^{1 / 2}$, it is easy to prove that

$$
\tilde{\angle} x q r=\tilde{\angle} p q r+O_{\mu}\left(\rho^{1 / 4}\right),
$$

where $O_{\mu}\left(\rho^{1 / 4}\right)$ is continuous in $\mu$. Therefore, the proof is completed in this case.

Assume that $t_{\rho} \geq \rho^{1 / 2}$. The definition of $t_{\rho}$ and the triangle inequality together imply

$$
d\left(\gamma\left(t_{\rho}\right), r\right) \geq d\left(p, \gamma\left(t_{\rho}\right)\right)+d(p, r)-2 \rho .
$$


If $t_{\rho}=d(p, q)$, we set $x:=q$. If $t_{\rho}<d(p, q)$, we find a number $t_{\rho}^{\prime} \in\left(t_{\rho}, \min \left\{t_{\rho}+\right.\right.$ $\rho, d(p, q)\})$ and set $x:=\gamma\left(t_{\rho}^{\prime}\right)$. Then, in either case, (4.3) implies

$$
d(x, r) \geq d(p, x)+d(p, r)-4 \rho,
$$

and hence, by (4.1),

$$
\tilde{\angle} p x r \leq O\left(\sqrt{\frac{d(p, r) \rho}{d(p, x) d(x, r)}}\right) .
$$

Here, it follows from (4.4) and $d(p, x) \geq \rho^{1 / 2}$ that $d(x, r) \geq d(p, r)+\rho^{1 / 2}-4 \rho \geq$ $d(p, r)$, so that

$$
\frac{d(p, r) \rho}{d(p, x) d(x, r)} \leq \rho^{1 / 2}
$$

Thus,

$$
\tilde{\angle} p x r \leq O\left(\rho^{1 / 4}\right) .
$$

If $x=q$, this completes the proof. If $x \neq q$, (4.5) implies $\tilde{\angle} p x r+\tilde{\angle} q x r \leq$ $\pi+O\left(\rho^{1 / 4}\right)$ and hence, by Lemma 4.3,

$$
\tilde{\angle} x q r \geq \tilde{\angle} p q r-O\left(\rho^{1 / 4}\right) .
$$

On the other hand, by Lemma 4.2 ,

$$
\tilde{\angle} x q r \leq \angle p q r+O\left(\epsilon_{\rho}\right) .
$$

This completes the proof.

4.3. Triangle comparison for limit spaces. For each $X \in \overline{\mathcal{M}(C, D)}$, by applying Corollary 2.1, there exists a (not necessarily unique) Radon measure $c_{\mathrm{abs}}^{X}$ over $X$ such that $\left(X, c_{\mathrm{abs}}^{X}\right)$ is a measured Gromov-Hausdorff limit of the class $\left\{\left(M, c_{\text {abs }}^{M}\right) \mid M \in \mathcal{M}(C, D)\right\}$.

Remark 4.1. Even if an open domain $D \subset X$ is isometric to a piece of $\mathbf{R}^{2}$, it may possibly have positive measure $c_{\text {abs }}^{X}(D)>0$. Let us give such an example. Assume first that a sequence $\left\{\left(M_{i}, c_{\text {abs }}^{M_{i}}\right)\right\}$ converges to $\left(X, c_{\text {abs }}^{X}\right)$ and a flat unit open disk $D_{i}=B\left(p_{i}, 1\right) \subset M$ tends to a flat unit open disk $D=B(p, 1) \subset X$ in this convergence, so that obviously, $c_{\text {abs }}^{M_{i}}\left(D_{i}\right)=c_{\text {abs }}^{X}(D)=0$. We then attach a small handle $H_{i}$ to $B\left(p_{i}, 1 / 2\right)$ which shrinks to the point $p \in D$ as $i \rightarrow \infty$. In this case, we still have $M_{i} \rightarrow X$; however $\left(M_{i}, c_{\text {abs }}^{M_{i}}\right)$ does not converge to $\left(X, c_{\text {abs }}^{X}\right)$ any more, because $c_{\text {abs }}\left(D_{i}\right) \geq-c\left(D_{i}\right)=4 \pi$. We may assume that $c_{\text {abs }}\left(D_{i}\right)$ tends to some number $a \geq 4 \pi$ as $i \rightarrow \infty$. Then, $\left(M_{i}, c_{\text {abs }}^{M_{i}}\right)$ converges to $\left(X, \hat{c}_{\text {abs }}^{X}\right)$, where $\hat{c}_{\text {abs }}^{X}$ is the Radon measure defined by

$$
\hat{c}_{\mathrm{abs}}^{X}(A):= \begin{cases}c_{\mathrm{abs}}^{X}(A) & \text { if } p \notin A, \\ c_{\mathrm{abs}}^{X}(A)+a & \text { if } p \in A\end{cases}
$$

for any Borel subset $A \subset X$.

Proof of the Triangle Comparison Theorem (1.5). Let $\varphi_{i}: M_{i} \rightarrow X, i=1,2, \ldots$, be measurable $\omega(1 / i)$-approximations from $M_{i} \in \mathcal{M}(C, D)$ to $X$ such that

$$
\lim _{i \rightarrow \infty} \int_{M_{i}} f \circ \varphi_{i} d c_{\mathrm{abs}}^{M_{i}}=\int_{X} f d c_{\mathrm{abs}}^{X}
$$


for any continuous function $f: X \rightarrow \mathbf{R}$. Find four points $p_{i}, q_{i}, r_{i}, s_{i} \in M_{i}$ such that $\varphi_{i}\left(p_{i}\right) \rightarrow p, \varphi_{i}\left(q_{i}\right) \rightarrow q, \varphi_{i}\left(r_{i}\right) \rightarrow r$, and $\varphi_{i}\left(s_{i}\right) \rightarrow s$ as $i \rightarrow \infty$, and set $\sigma_{i}:=p_{i} s_{i}$, $\tau_{i}:=s_{i} q_{i}$. By replacing with a subsequence, it may be assumed that there are two minimal segments $\sigma$ from $p$ to $s$ and $\tau$ from $s$ to $q$ in $X$ such that $\varphi_{i}\left(\sigma_{i}\right)$ and $\varphi_{i}\left(\tau_{i}\right)$ both tend to $\sigma$ and $\tau$ respectively. Applying Lemma 4.4 one has for any fixed $\rho>0$,

$$
\begin{aligned}
& \angle p_{i} s_{i} r_{i} \geq \tilde{\angle} p_{i} s_{i} r_{i}-O\left(c_{\mathrm{abs}}^{M_{i}}\left(K\left(r_{i}, \sigma_{i}\right)-B\left(p_{i}, \rho\right)\right)\right)-O_{\mu_{i}}\left(\rho^{1 / 4}\right), \\
& \angle q_{i} s_{i} r_{i} \geq \tilde{\angle} q_{i} s_{i} r_{i}-O\left(c_{\mathrm{abs}}^{M_{i}}\left(K\left(r_{i}, \tau_{i}\right)-B\left(q_{i}, \rho\right)\right)\right)-O_{\mu_{i}}\left(\rho^{1 / 4}\right),
\end{aligned}
$$

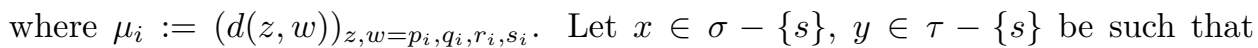
$d(s, x), d(s, y) \ll \rho$, and let $x_{i} \in \sigma_{i}, y_{i} \in \tau_{i}$ be such that $\varphi_{i}\left(x_{i}\right) \rightarrow x$ and $\varphi_{i}\left(y_{i}\right) \rightarrow y$ as $i \rightarrow \infty$. Then, Lemma 4.4 implies that for all sufficiently large $i$,

$$
\angle p_{i} s_{i} q_{i}=\angle x_{i} s_{i} y_{i} \geq \tilde{L} x_{i} s_{i} y_{i}-O\left(c_{\mathrm{abs}}^{M_{i}}\left(B\left(s_{i}, \rho\right)\right)\right)-O_{\nu_{i}}\left(\rho^{1 / 4}\right),
$$

where $\nu_{i}:=\left(d\left(x_{i}, y_{i}\right), d\left(y_{i}, s_{i}\right), d\left(s_{i}, x_{i}\right)\right)$. Therefore,

$$
\begin{aligned}
2 \pi & \geq \angle p_{i} s_{i} r_{i}+\angle q_{i} s_{i} r_{i}+\angle p_{i} s_{i} q_{i} \\
& \geq \tilde{\angle} p_{i} s_{i} r_{i}+\tilde{\angle} q_{i} s_{i} r_{i}+\tilde{L} x_{i} s_{i} y_{i}-O\left(c_{\mathrm{abs}}^{M_{i}}\left(K\left(r_{i}, \sigma_{i}\right)-B\left(p_{i}, \rho\right)\right)\right) \\
& -O\left(c_{\mathrm{abs}}^{M_{i}}\left(K\left(r_{i}, \tau_{i}\right)-B\left(q_{i}, \rho\right)\right)\right)-O\left(c_{\mathrm{abs}}^{M_{i}}\left(B\left(s_{i}, \rho\right)\right)\right)-O_{\mu_{i}, \nu_{i}}\left(\rho^{1 / 4}\right) .
\end{aligned}
$$

Since $\tilde{\angle} x_{i} s_{i} y_{i} \rightarrow \tilde{L} x s y=\pi$, taking $\rho \rightarrow 0$ after $i \rightarrow \infty$ yields

$$
\tilde{\angle} p s r+\tilde{\angle} q s r \leq \pi+O\left(c_{\text {abs }}^{X}(K(r, \sigma)-\{p\})\right)+O\left(c_{\text {abs }}^{X}(K(r, \tau)-\{q\})\right) .
$$

Now, if $q \notin K(r, \sigma)$ and $p \notin K(r, \tau)$, then

$$
\tilde{\angle} p s r+\tilde{\angle} q s r \leq \pi+O\left(c_{\mathrm{abs}}^{X}(K(r, \sigma \cup \tau)-\{p, q\})\right) .
$$

If $q \in K(r, \sigma)$, there are a point $z \in \sigma$ and a minimal segment $z r \ni q$, so that $\tau \cup q r$ is a minimal segment and $\tilde{\angle} q s r=0$; in particular,

$$
\tilde{\angle} p s r+\tilde{\angle} q s r \leq \pi \text {. }
$$

If $p \in K(r, \tau)$, then $\tilde{\angle} p s r=0$ and hence

$$
\tilde{\angle} p s r+\tilde{\angle} q s r \leq \pi \text {. }
$$

Thus, in either case, by Lemma 4.3 we have

$$
\tilde{\angle} s p r \geq \tilde{\angle} q p r-O\left(c_{\mathrm{abs}}^{X}(K(r, \sigma \cup \tau)-\{p, q\})\right) .
$$

The same discussion leads to

$$
\tilde{\angle} s p r \geq \tilde{\angle} q p r-O\left(c_{\mathrm{abs}}^{X}(K(\{p, q\}, \gamma)-\{r\})\right)
$$

for some minimal segment $\gamma$ joining $r$ and $s$. This completes the proof.

\subsection{Angle between minimal segments in the limit spaces.}

Definition 4.1 (Angle). Let $X \in \overline{\mathcal{M}(C, D)}, p, q, r \in X, \gamma:=p q$, and $\sigma:=p r$. Since $\lim _{\rho \rightarrow 0} c_{\mathrm{abs}}^{X}(B(p, \rho)-\{p\})=0$, the Triangle Comparison Theorem (1.5) proves the existence of the limit

$$
\lim _{s, t>0, s, t \rightarrow 0} \tilde{\angle} \gamma(s) p \sigma(t),
$$

which we call the angle at $p$ between $\gamma$ and $\sigma$, and denote by $\angle(\gamma, \sigma)$ or $\angle q p r$.

The following proposition, analogous to the Toponogov comparison theorem, is a direct consequence of the definition of the angle. 
Proposition 4.1. For any triangle $\triangle p q r$ in $X \in \overline{\mathcal{M}(C, D)}$, we have

$$
\angle q p r \geq \tilde{\angle} q p r-O\left(c_{\mathrm{abs}}^{X}(B(p, 2(d(p, q)+d(p, r)))-\{p\})\right) .
$$

We have the following properties of the angle, the proofs of which are slightly modified versions of those of 2.8 in [6] and are omitted.

Lemma 4.5. Let $X \in \overline{\mathcal{M}(C, D)}$.

(1) For any $p, q, r, s \in X$ with $p \in q r-\{q, r\}$,

$$
\pi \leq \angle q p s+\angle r p s \leq \pi+O\left(c_{\mathrm{abs}}^{X}(\{p\})\right) .
$$

(2) If two sequences of segments $p_{i} q_{i}$ and $p_{i} r_{i}$ respectively tend to $p q$ and $p r$ in $X$ as $i \rightarrow \infty$, then

$$
\liminf _{i \rightarrow \infty} \angle q_{i} p_{i} r_{i} \geq \angle q p r-O\left(c_{\mathrm{abs}}^{X}(\{p\})\right) .
$$

\section{Structure of the SPACE of Directions}

Throughout this section, let $X \in \overline{\mathcal{M}(C, D)}$ be a limit space. In this section, we will define the space of directions at a point in $X$ and prove that it is a finite disjoint union of points and circles (see Theorem 5.2).

5.1. Foundation of the space of directions. Since we have the triangle inequality for the angles between minimal segments from a common point $p \in X$ (see Theorem 1 of Chapter II of [4]), the angle is a pseudo-distance function on the set of minimal segments $\{p q\}_{q \in X}$, so that the relation between minimal segments from $p$ that two have angle zero is an equivalence relation, and the quotient space, say $\Sigma_{p}^{\prime}$, of $\{p q\}_{p \in X}$ modulo this equivalence relation becomes a metric space.

Definition 5.1 (Space of Directions and Tangent Cone). The metric completion of $\Sigma_{p}^{\prime}$ for a point $p \in X$ is called the space of directions at $p$ and denoted by $\Sigma_{p}$ or $\Sigma_{p} X$. We call each element of $\Sigma_{p}$ a direction at $p$. Denote by $v_{p q}$ the direction at $p$ which is the equivalence class represented by a minimal segment $p q$ in $X$. The tangent cone $K_{p}$ at $p$ is defined to be the Euclidean cone over $\Sigma_{p}$, where the Euclidean cone 'cone $Y$ ' over a metric space $Y$ is defined to be the quotient space $Y \times[0,+\infty) / Y \times\{0\}$ with the metric defined by

$$
d((v, s),(w, t)):=\sqrt{s^{2}+t^{2}-2 s t \cos \min \{d(v, w), \pi\}}
$$

for $(v, s),(w, t) \in$ cone $Y$. Denote by $o$ the point of $K_{p}$ corresponding to $\Sigma_{p} \times\{0\}$ and call it the vertex of $K_{p}$.

For a metric space $Y$ and a number $\lambda>0$ we denote by $\lambda Y$ the space $Y$ with distance function multiplied by $\lambda$-times.

Proposition 5.1. For any $p \in X$ and $\epsilon>0$ we have

$$
\beta_{\Sigma_{p}}(\epsilon) \leq \operatorname{const}_{C}\left(1+\epsilon^{-1}\right) .
$$

In particular, the space of directions $\Sigma_{p}$ at any point $p \in X$ is compact and satisfies

$$
\mathcal{H}^{1}\left(\Sigma_{p}\right) \leq \operatorname{const}_{C}
$$


Proof. Let $p \in M$ and $0<\epsilon \ll \pi$ be both fixed. To prove the first assertion, it suffices to prove that $\beta_{\Sigma_{p}^{\prime}}(\epsilon) \leq$ const $_{C}\left(1+\epsilon^{-1}\right)$. We find a maximal net $N \subset \Sigma_{p}^{\prime}$ such that $\angle(v, w) \geq \epsilon$ for any different $v, w \in N$. Notice here that $N$ may possibly be an infinite net. Assign a minimal segment $\gamma_{v}$ to each $v \in \Sigma_{p}^{\prime}$ in such a way that the direction of $\gamma_{v}$ is $v$. It follows from the definition of the angle that there is a $\rho_{v, w}>0$ for any different $v, w \in N$ such that $\tilde{L}\left(\gamma_{v}(s), \gamma_{w}(t)\right) \geq 0.99 \epsilon$ for any $s, t \in\left(0, \rho_{v, w}\right]$. Hence, by remarking $\epsilon \ll \pi$, there exists a $\rho>0$ depending on any finite subnet $\hat{N}$ of $N$ such that

$$
d\left(\gamma_{v}(s), \gamma_{w}(t)\right) \geq \epsilon \rho / 2
$$

for any $s, t \in(0,2 \rho]$ and for any different $v, w \in \hat{N}$. This implies that

$$
\beta_{\bar{B}(p, 2 \rho)}(\epsilon \rho / 2) \geq \beta_{\bar{B}(p, 2 \rho)-B(p, \rho)}(\epsilon \rho / 2) \geq\left[2 \epsilon^{-1}\right] \cdot \# \hat{N} \geq\left(2 \epsilon^{-1}-1\right) \cdot \# \hat{N} .
$$

For any $\delta>0$ there exist an $M \in \mathcal{M}(C, D)$ and a $\delta$-approximation $\varphi: X \rightarrow M$. We thus have $\beta_{\bar{B}(\varphi(p), 2 \rho+\delta)}(\epsilon \rho / 2-\delta) \geq\left(2 \epsilon^{-1}-1\right) \cdot \# \hat{N}$, where, if $\delta$ is taken to be small enough, then

$$
\beta_{\bar{B}(\varphi(p), 3 \rho)}(\epsilon \rho / 3) \geq\left(2 \epsilon^{-1}-1\right) \cdot \# \hat{N} .
$$

On the other hand, since the total absolute curvature is an invariant up to scaling metric, one has $c_{\mathrm{abs}}\left(\rho^{-1} M\right) \leq C$, so that applying Lemma 3.3 to $B\left(\varphi(p), 3 ; \rho^{-1} M\right)$ yields

$$
\beta_{\bar{B}(\varphi(p), 3 \rho)}(\epsilon \rho / 3)=\beta_{\bar{B}\left(\varphi(p), 3 ; \rho^{-1} M\right)}(\epsilon / 3) \leq \operatorname{const}_{C}\left(1+\epsilon^{-2}\right) .
$$

Therefore we obtain $\# \hat{N} \leq$ const $_{C}\left(1+\epsilon^{-1}\right)$. Since $\hat{N}$ is any finite subnet of $N$, this proves the first assertion.

Since $\beta_{\Sigma_{p}}(\epsilon)<+\infty$ means the precompactness of $\Sigma_{p}$, the second assertion directly follows from the first (see $\S 2.3$ ).

Remark 5.1. For Alexandrov spaces, the compactness of the space of directions is implied by only the Alexandrov convexity (i.e., the Triangle Comparison Theorem under $\left.c_{\mathrm{abs}}^{X} \equiv 0\right)$. On the other hand, that is not true in our case as seen in $\S 9$.

Proposition 5.2. For any fixed point $p \in X$, the pointed space $\left(\rho^{-1} X, p\right)$ tends to $\left(K_{p}, o\right)$ as $\rho \rightarrow 0$.

Proof. The proposition is proved by the same discussion as in 7.8.1 of [6] using the compactness of the space of directions and the triangle comparison theorem.

From Propositions 5.1 and 5.2, we see that the tangent cone $K_{p}$ at any $p \in X$ is a complete locally compact intrinsic metric space of Hausdorff dimension $\leq 2$. Define a Radon measure $c_{\text {abs }}^{K_{p}}$ over $K_{p}$ by

$$
c_{\mathrm{abs}}^{K_{p}}(A):= \begin{cases}0 & \text { if } o \notin A, \\ c_{\mathrm{abs}}^{X}(\{p\}) & \text { if } o \in A\end{cases}
$$

for any Borel subset $A \subset K_{p}$. Then, since $\lim _{\rho \rightarrow 0} c_{\text {abs }}^{X}(B(p, \rho)-\{p\})=0$, we have that $\left(\rho^{-1} X, p, c_{\mathrm{abs}}^{X}\right)$ converges to $\left(K_{p}, o, c_{\mathrm{abs}}^{K_{p}}\right)$ as $\rho \rightarrow 0$ in the sense of the measured and pointed Gromov-Hausdorff convergence. Therefore, $\left(K_{p}, c_{\mathrm{abs}}^{K_{p}}\right)$ satisfies the Triangle Comparison Theorem (1.5).

Proposition 5.3. The space of directions $\Sigma_{p}$ at any point $p \in X$ is a finite disjoint union of points, circles, and compact arcs. 
Proof. Since $\Sigma_{p}$ is compact, $K_{p}-\{o\}$ has at most finitely many connected components. Each component $A$ of $K_{p}-\{o\}$ satisfies $c_{\text {abs }}^{K_{p}}(A)=0$, i.e., $A$ is nonnegatively curved in the sense of Alexandrov and hence each component of $\Sigma_{p}$ has curvature $\geq 1$ locally (see 4.2.3 of [6]). By recalling Proposition 5.1, this completes the proof.

Lemma 5.1. For any $u, v, w \in \Sigma_{p}, p \in X$, we have

$$
\angle(u, v)+\angle(v, w)+\angle(w, u) \leq 2 \pi+O\left(c_{\mathrm{abs}}^{X}(\{p\})\right) .
$$

Proof. For simplicity, let $\epsilon:=c_{\mathrm{abs}}^{X}(\{p\})$. Then, $\epsilon=c_{\mathrm{abs}}^{K_{p}}\left(K_{p}\right)=c_{\mathrm{abs}}^{K_{p}}(\{o\})$. Proposition 4.1 implies that $\angle \bar{u} \bar{v} \bar{w} \geq \angle \bar{u} \bar{v} \bar{w}-O(\epsilon)$, where $\bar{u}:=(u, 1), \bar{v}:=(v, 1), \bar{w}:=$ $(w, 1) \in K_{p}$. On the other hand, $\angle \bar{u} \bar{v} \bar{w} \leq \angle o \bar{v} \bar{u}+\angle o \bar{v} \bar{w}=\pi-(\angle(u, v)+\angle(v, w)) / 2$. Therefore,

$$
\pi-(\angle(u, v)+\angle(v, w)) / 2 \geq \tilde{L} \bar{u} \bar{v} \bar{w}-O(\epsilon)
$$

as well as

$$
\begin{aligned}
& \pi-(\angle(v, w)+\angle(w, u)) / 2 \geq \tilde{L} \bar{v} \bar{w} \bar{u}-O(\epsilon), \\
& \pi-(\angle(w, u)+\angle(u, v)) / 2 \geq \tilde{L} \bar{w} \bar{u} \bar{v}-O(\epsilon) .
\end{aligned}
$$

Adding up these three completes the proof.

5.2. Strainer. In order to more study the space of directions, we need the notion of strainer introduced in [6].

Definition 5.2. A point $p \in X$ is said to be $(n, \delta)$-strained, $n \in \mathbf{N}, \delta>0$, if there exist points $q_{i} \in X, i= \pm 1, \ldots, \pm n$, such that

$$
\tilde{\angle} q_{i} p q_{j}> \begin{cases}\pi / 2-\delta, & \text { if } i \neq \pm j, \\ \pi-\delta, & \text { if } i=-j .\end{cases}
$$

Here, the sequence $\left\{q_{i}\right\}_{i= \pm 1, \ldots, \pm n}$ is called an $(n, \delta)$-strainer at $p$.

Remark 5.2. Assume that $c_{\mathrm{abs}}(B(p, R)) \leq \delta$ for a point $p \in X$ and two numbers $\delta, R>0$. Then, there exists an $(n, O(\delta))$-strainer $\left\{q_{i}\right\}$ at $p$ such that $d\left(p, q_{i}\right) \leq R / 2$ for each $i$ if and only if there exists $\left\{v_{i}\right\}_{i= \pm 1, \ldots, \pm n} \subset \Sigma_{p}$ such that

$$
\angle\left(v_{i}, v_{j}\right)> \begin{cases}\pi / 2-O(\delta), & \text { if } i \neq \pm j, \\ \pi-O(\delta), & \text { if } i=-j .\end{cases}
$$

Definition 5.3. A map $f$ from a metric space $Y$ to a metric space $Z$ is said to be $\delta$-almost isometric, $\delta>0$, if

$$
\left|\frac{d(f(p), f(q))}{d(p, q)}-1\right| \leq \delta \quad \text { for any } p, q \in Y
$$

Remarking that $X$ has Hausdorff dimension $\leq 2$, we have the following theorem in the same way as in $\S 5$ of [6]. Here, we omit the proof.

Theorem 5.1. Assume that a point $p \in X$ is an $(n, \delta)$-strained point with a strainer $\left\{q_{i}\right\}_{i= \pm 1, \ldots, \pm n}$ for given numbers $n \in \mathbf{N}$ and $\delta>0$ and that

$$
c_{\mathrm{abs}}^{X}\left(B\left(p, 2 \max _{i} d\left(p, q_{i}\right)\right)\right)<\delta .
$$

Then, we have the following (1) and (2). 
(1) There exists a universal constant $\delta_{0}>0$ such that, if $\delta \leq \delta_{0}$, we have $n \leq 2$.

(2) Assume $n=2$ and let $f: B(p, \rho) \rightarrow \mathbf{R}^{2}$ for $a \rho>0$ be the map defined by $f(x):=\left(d\left(q_{1}, x\right), d\left(q_{2}, x\right)\right)$ for any $x \in B(p, \rho)$. Then, there exists a universal constant $\epsilon_{0}>0$ such that, if $\epsilon:=\delta+\rho / \min _{i} d\left(p, q_{i}\right) \leq \epsilon_{0}$, the map $f$ is an $\omega(\epsilon)$-almost isometric into homeomorphism and its image $f(B(p, \rho))$ is an open subset of $\mathbf{R}^{2}$.

The following lemma is important for the proof of Theorem 5.1 and will be used later.

Lemma 5.2. Assume that $\tilde{\angle} p r q \geq \pi-\delta, \max \{d(r, s) / d(p, r), d(r, s) / d(q, r)\} \leq \delta$, and $c_{\mathrm{abs}}^{X}(B(r, 2 \max \{d(p, r), d(q, r)\})-\{p\}) \leq \delta$ for four different points $p, q, r, s \in$ $X$ and for a number $\delta>0$. Then, we have

$$
\begin{gathered}
\tilde{\angle} p r s+\tilde{\angle} q r s=\pi+\omega(\delta), \\
\angle p r s=\tilde{\angle} p r s+\omega(\delta), \quad \angle q r s=\tilde{\angle} q r s+\omega(\delta) .
\end{gathered}
$$

In addition, if $|d(p, r)-d(p, s)| / d(r, s) \leq \delta$, then

$$
\tilde{\angle p r s}, \tilde{\angle} q r s, \angle p r s, \angle q r s=\frac{\pi}{2}+\omega(\delta) \text {. }
$$

The proof of the lemma is also omitted.

5.3. Nonexistence of the boundary of the space of directions. The following lemma is essential to prove that the space of directions $\Sigma_{p}$ at any point $p \in X$ contains no arc as a component.

Lemma 5.3. There exists a $\delta>0$ such that, if a sequence of pointed Riemannian 2 -manifolds $\left(M_{i}, p_{i}\right)$ satisfies $c_{\mathrm{abs}}^{M_{i}}\left(B\left(p_{i}, 1\right)\right) \leq \delta$ for every $i$, then it never converges to the half-plane $(\mathbf{R} \times[0,+\infty), o), o:=(0,0)$.

Proof. Suppose that such a sequence $\left(M_{i}, p_{i}\right)$ converges to the half plane $(H, p):=$ $(\mathbf{R} \times[0,+\infty), o)$. Let $q:=(1,0) \in H$ and $\left\{x_{s}\right\}:=\partial B(p, s) \cap \partial B(q, 1) \subset H$ for $0<$ $s<1$. We find a $d_{i}$-approximation $\varphi_{i}:(B(p, 10), p) \rightarrow\left(B\left(p_{i}, 10\right), p_{i}\right)$, where $d_{i} \rightarrow 0$, and put $q_{i}:=\varphi_{i}(q)$. Since $x_{s}$ is a $(2, \omega(s))$-strained point with strainer containing $\{p, q\}$, the point $\varphi_{i}\left(x_{s}\right)$ for each $i$ is a $\left(2, \omega\left(s+d_{i} / s\right)\right)$-strained point with the strainer containing $\left\{p_{i}, q_{i}\right\}$. Find a number $\rho>0$ and assume that $d_{i} \ll \rho \ll s \ll 1$. Then, applying Theorem 5.1 yields that the map $f_{i}:=\left(d\left(p_{i}, \cdot\right), d\left(q_{i}, \cdot\right)\right)$ from $B\left(\varphi_{i}\left(x_{s}\right), \rho\right)$ to $\mathbf{R}^{2}$ is an $\omega\left(s+d_{i} / s+\rho / s\right)$-almost isometric into homeomorphism and its image is an open subset of $\mathbf{R}^{2}$. Since $\left|d\left(p_{i}, q_{i}\right)-1\right| \leq \omega\left(d_{i}\right)$ and $\left|(s, 1)-f_{i}\left(\varphi_{i}\left(x_{s}\right)\right)\right| \leq \omega\left(d_{i}\right)$, one has $\left(s, d\left(p_{i}, q_{i}\right)\right) \in B\left(f_{i}\left(\varphi_{i}\left(x_{s}\right)\right), \omega\left(d_{i}\right)\right) \subset f_{i}\left(B\left(\varphi_{i}\left(x_{s}\right), \rho\right)\right)$ and hence there is a point $x_{s, i} \in B\left(\varphi_{i}\left(x_{s}\right), \rho\right)$ such that $f_{i}\left(x_{s, i}\right)=\left(s, d\left(p_{i}, q_{i}\right)\right)$. We now prove that

$$
\partial B\left(p_{i}, s\right) \cap \partial B\left(q_{i}, d\left(p_{i}, q_{i}\right)\right)=\left\{x_{s, i}\right\} .
$$

In fact, $x_{s, i} \in \partial B\left(p_{i}, s\right) \cap \partial B\left(q_{i}, d\left(p_{i}, q_{i}\right)\right)$ is trivial. Let $y_{i}$ be any point in $\partial B\left(p_{i}, s\right) \cap$ $\partial B\left(q_{i}, d\left(p_{i}, q_{i}\right)\right)$ and find $y \in H$ in such a way that $d\left(\varphi_{i}(y), y_{i}\right) \leq d_{i}$. Since $d(p, y)=$ $s+\omega\left(d_{i}\right)$ and $d(q, y)=d\left(p_{i}, q_{i}\right)+\omega\left(d_{i}\right)=1+\omega\left(d_{i}\right)$, one has $d\left(x_{s}, y\right) \leq \omega\left(d_{i}\right)$ and hence $d\left(\varphi_{i}\left(x_{s}\right), y_{i}\right) \leq \omega\left(d_{i}\right)$, which together with $d_{i} \ll \rho$ implies that $y_{i}$ is contained in $B\left(\varphi_{i}\left(x_{s}\right), \rho\right)$. Since $f_{i}\left(y_{i}\right)=\left(s, d\left(p_{i}, q_{i}\right)\right)$, we have $y_{i}=x_{s, i}$, so that (5.1) has been proved.

Since, by $d_{i} \ll 1, \partial B\left(q_{i}, d\left(p_{i}, q_{i}\right)\right)$ contains no critical points of the distance function to $q_{i}$, it is a compact 1-manifold without boundary, i.e., a finite disjoint union of circles. The component, say $S$, of $\partial B\left(q_{i}, d\left(p_{i}, q_{i}\right)\right)$ containing $x_{s, i}$ splits 
into the two parts $S \cap B\left(p_{i}, s\right)$ and $S \cap\left(X-B\left(p_{i}, s\right)\right)$ whose joint consists of the only one point $x_{s, i}$. This is a contradiction.

Theorem 5.2. The space of directions $\Sigma_{p}$ at any point $p \in X$ is a finite disjoint union of points and circles.

Proof. It suffices to prove that $\Sigma_{p}$ has no component homeomorphic to a closed line segment. Suppose the contrary and let $v \in \Sigma_{p}$ be a terminal point of a component homeomorphic to a line segment. Then, by setting $\bar{v}:=(v, 1) \in K_{p}$, the pointed space $\left(\rho^{-1} K_{p}, \bar{v}\right)$ tends to the half plane $(H, o):=(\mathbf{R} \times[0,+\infty), o)$ as $\rho \rightarrow 0$. By Proposition 5.2, there are a positive number $R_{\rho} \nearrow+\infty$ and an $\omega_{p}(\rho)$-approximation $\varphi_{\rho}: B\left(o, R_{\rho} ; K_{p}\right) \rightarrow B\left(p, R_{\rho} ; \rho^{-1} X\right)$. Let $q_{\rho}:=\varphi_{\rho}(\bar{v})$. Since $\left(\rho^{-1} X, q_{\rho}\right)$ tends to $\left(K_{p}, \bar{v}\right)$, finding a positive number $R_{\rho}^{\prime}$ slowly tending to $+\infty$ as $\rho \rightarrow 0$ one has

$$
\lim _{\rho \rightarrow 0}\left(\rho^{-1} R_{\rho}^{\prime} X, q_{\rho}\right)=\lim _{\rho \rightarrow 0}\left(R_{\rho}^{\prime} K_{p}, \bar{v}\right)=(H, o) .
$$

Besides, it follows from $d\left(p, q_{\rho}\right) / \rho=1+\omega_{p}(\rho)$ that $c_{\mathrm{abs}}^{X}\left(B\left(q_{\rho}, \rho R_{\rho}^{\prime-1}\right)\right)$ tends to 0 as $\rho \rightarrow 0$. Find a sequence of manifolds $M_{i} \in \mathcal{M}(C, D)$ tending to $X$ and an $\omega(1 / i)$ approximation $f_{i}: X \rightarrow M_{i}$. There exists a number $i(\rho)$ for each $\rho>0$ such that the pointed space $\left(\rho^{-1} R_{\rho}^{\prime} M_{i(\rho)}, f_{i(\rho)}\left(q_{\rho}\right)\right)$ tends to $(H, o)$ and $c_{\text {abs }}\left(B\left(f_{i(\rho)}\left(q_{\rho}\right), \rho R_{\rho}^{\prime}{ }^{-1}\right)\right)$ to 0 as $\rho \rightarrow 0$, which contradicts the previous lemma.

Remark 5.3. Although, by the definition, the angle $\angle(v, w)$ between any $v, w \in \Sigma_{p}$ is $\leq \pi$, yet the minimal length of curves in $\Sigma_{p}$ joining two directions may possibly exceed $\pi$. If two directions $v, w \in \Sigma_{p}$ satisfy $\angle(v, w)<\pi$, then $v$ and $w$ are contained in a common component of $\Sigma_{p}$ and $\angle(v, w)$ is just the minimal length of curves joining $v$ and $w$ in $\Sigma_{p}$. Therefore, if $\hat{\angle}$ denotes the intrinsic metric of $\Sigma_{p}$ induced from $\angle$, it may exceed $\pi$ and we have

$$
\angle(v, w)=\min \{\hat{\angle}(v, w), \pi\} \quad \text { for any } v, w \in \Sigma_{p} .
$$

Notice here that if two directions $v, w \in \Sigma_{p}$ are contained in different components, we have $\angle(v, w)=\pi$ and $\hat{L}(v, w)=+\infty$.

The following proposition is a direct consequence of Theorem 5.2, Remark 5.3 and Lemma 5.1.

\section{Proposition 5.4.}

(1) The length of each circle component of $\Sigma_{p}$ at any $p \in X$ is $\leq 2 \pi+O\left(c_{\mathrm{abs}}^{X}(\{p\})\right)$.

(2) There exists a universal constant $\delta>0$ such that, if $c_{\mathrm{abs}}^{X}(\{p\}) \leq \delta$ for a point $p \in X$, the space of directions $\Sigma_{p}$ at $p$ has at most two connected components, and if it has exactly two components, the diameter of each component is $\leq O\left(c_{\mathrm{abs}}^{X}(\{p\})\right)$.

\section{Topological Structure of the limit SPACES}

In this section we will prove the Topological Structure Theorem (1.2). Throughout this section, let $X \in \overline{\mathcal{M}(C, D)}$ be a limit space. 
6.1. Construction of local polar coordinate. The main purpose of this subsection is to construct a bi-Lipschitz homeomorphism (a so-called local polar coordinate) between an annulus and a small neighborhood of a circle component of the distance sphere $\partial B(p, \rho)$ centered at a point $p \in X$ and with a small radius $\rho>0$ (see Lemma 6.2).

Lemma 6.1. For any $p, x \in X$ and $\lambda>1$ we have the following:

(1) for any $\rho \in(d(p, x), \lambda d(p, x)]$ there exists a point $q \in \partial B(p, \rho)$ such that $\{p, q\}$ is a $\left(1, \omega_{p, \lambda}(d(p, x))\right)$-strainer at $x$;

(2) if $x$ is not an isolated point of $\partial B(p, d(p, x))$, then $x$ is a $\left(2, \omega_{p, \lambda}(d(p, x))\right)$ strained point with strainer $\{p, q, r, s\}$ for some $r, s \in X$ and the $q$ in (1).

Proof. (1): Let $p, x \in X, \lambda>1$, and $\rho \in(d(p, x), \lambda d(p, x)]$. By Proposition 5.2, supposing $d(p, x)$ is small enough against $p$ and $\lambda$, there is a point $q^{\prime} \in X$ such that $\lambda d(p, x) \leq d\left(p, q^{\prime}\right) \leq 2 \lambda d(p, x)$ and $\tilde{\angle} p x q^{\prime} \geq \pi-\omega_{p, \lambda}(d(p, x))$. If one finds a point $q \in x q^{\prime}$ with $d(p, q)=\rho$, applying Theorem 1.5 yields

$$
\tilde{\angle} p x q \geq \tilde{\angle} p x q^{\prime}-\omega_{p}\left(d\left(p, q^{\prime}\right)\right) \geq \pi-\omega_{p, \lambda}(d(p, x)) .
$$

(2): Assume that $x$ is not an isolated point of $\partial B(p, d(p, x))$. Then, there is a sequence $\left\{x_{i}\right\}$ of points in $\partial B(p, d(p, x))$ tending to $x$ such that $x_{i} \neq x$ for any $i$. Let $r^{\prime}:=x_{i}$ for a sufficiently large $i$. Since $\tilde{\angle p x q} \geq \pi-\omega_{p, \lambda}(d(p, x))$ and $d\left(p, r^{\prime}\right)=$ $d(p, x)$, applying Lemma 5.2 yields that $\angle p x r^{\prime}, \angle q x r^{\prime}=\pi / 2+\omega_{p, \lambda}(d(p, x))$. This and Lemma 5.4 together show that $\Sigma_{x}$ is isometric to the circle of length $2 \pi+$ $\omega_{p, \lambda}(d(p, x))$ provided $x$ is close enough to $p$. One can find a point $s^{\prime} \in X$ such that $\angle r^{\prime} x s^{\prime} \geq \pi-\omega_{p, \lambda}(d(p, x))$ and $\angle p x s^{\prime}, \angle q x s^{\prime}=\pi / 2+\omega_{p, \lambda}(d(p, x))$. If two points $s \in$ $x s^{\prime}$ and $r \in x r^{\prime}$ are taken to be close enough to $x$, we have $\tilde{\angle} r x s \geq \pi-\omega_{p, \lambda}(d(p, x))$, so that Lemma 5.2 proves that $\{p, q, r, s\}$ is a $\left(2, \omega_{p, \lambda}(d(p, x))\right)$-strainer at $x$.

Lemma 6.2. If a number $\rho>0$ is small enough against a given point $p \in X$, then

(1) $\partial B(p, \rho)$ is a finite disjoint union of points and rectifiable circles;

(2) for each circle component $\Gamma$ of $\partial B(p, \rho)$ there exist a neighborhood $U$ of $\Gamma$, a number $\epsilon>0$, and a map $\psi: U \rightarrow \Gamma$ such that $f:=(d(p, \cdot), \psi): U \rightarrow$ $(\rho-\epsilon, \rho+\epsilon) \times \Gamma$ is a bi-Lipschitz homeomorphism.

Proof. (1): Let $\Gamma$ be a connected component of $\partial B(p, \rho)$ such that $\Gamma$ does not consist of only one isolated point of $\partial B(p, \rho)$. To prove (1), it suffices to show that $\Gamma$ is a rectifiable circle. Lemma 6.1(2) implies that for any point $x \in \Gamma$ there are $q_{x}, r_{x}, s_{x} \in X$ such that $\left\{p, q_{x}, r_{x}, s_{x}\right\}$ is a $\left(2, \omega_{p}(\rho)\right)$-strainer at $x$. If $\rho$ is small enough against $p$, by Theorem 5.1, there are a neighborhood $U_{x}$ at $x$ and a number $\epsilon_{x}>0$ such that $\varphi_{x}:=\left(d(p, \cdot), d\left(r_{x}, \cdot\right)\right): U_{x} \rightarrow I\left(\rho, \epsilon_{x}\right) \times I\left(d\left(r_{x}, x\right), \epsilon_{x}\right)$ is an $\omega_{p}(\rho)$ almost isometric homeomorphism, where $I(a, d):=(a-d, a+d) \subset \mathbf{R}$ for $a \in \mathbf{R}$ and $d>0$. Hence, $\partial B(p, \rho) \cap U_{x}=\varphi_{x}^{-1}\left(\{\rho\} \times I\left(d\left(r_{x}, x\right), \epsilon_{x}\right)\right)$ for any $x \in \Gamma$ is a rectifiable open arc. Since $\Gamma$ is compact, it is a rectifiable circle.

(2): Under the same notation as in the proof of (1), there are finitely many points $x_{1}, \ldots, x_{k}$ in a circle component $\Gamma$ of $\partial B(p, \rho)$ such that $\Gamma \subset \bigcup_{i} U_{x_{i}}$. Gluing the almost isometric homeomorphisms $\varphi_{x_{i}}, i=1, \ldots, k$, by using partition of unity, we obtain the desired bi-Lipschitz homeomorphism $f$ from some tubular neighborhood $U$ of $\Gamma$ to $(\rho-\epsilon, \rho+\epsilon) \times \Gamma, \epsilon:=\min _{i} \epsilon_{x_{i}}$, with the property that $f=(d(p, \cdot), \psi)$ for some Lipschitz map $\psi: U \rightarrow \Gamma$. Since this gluing process to construct $f$ is by a standard discussion, we omit the details. 
Remark 6.1. Although we can prove that $f$ is locally almost isometric in the proof of Lemma 6.2 , yet it is not necessarily almost isometric globally, because a minimal segment joining two points in $U$ may not be contained in $U$.

Lemma 6.3. For any $p \in X, R>0$, and $0<\lambda \leq 1$, there exists a family of maps $\left\{\Phi_{\rho, \rho^{\prime}}: \partial B(p, \rho) \rightarrow \partial B\left(p, \rho^{\prime}\right)\right\}_{\rho, \rho^{\prime} \in[\lambda R, R]}$ such that

$$
d\left(x, \Phi_{\rho, \rho^{\prime}}(x)\right)=\left(1+\omega_{p, \lambda}(R)\right)\left|\rho-\rho^{\prime}\right|
$$

for any $\rho, \rho^{\prime} \in[\lambda R, R]$ and $x \in \partial B(p, \rho)$.

Proof. If $\rho \geq \rho^{\prime}$, we find a point $\Phi_{\rho, \rho^{\prime}}(x) \in p x \cap \partial B\left(p, \rho^{\prime}\right)$ and have

$$
d\left(x, \Phi_{\rho, \rho^{\prime}}(x)\right)=\left|\rho-\rho^{\prime}\right| .
$$

If $\rho<\rho^{\prime}$, by Lemma $6.1(1)$, there is a point $q \in \partial B\left(p, \rho^{\prime}\right)$ such that $\tilde{\angle} p x q \geq$ $\pi-\omega_{p, \lambda}(R)$. Defining $\Phi_{\rho, \rho^{\prime}}(x):=q$ completes the proof.

The following corollary is a direct consequence of Lemma 6.3.

Corollary 6.1. For any $p \in X$ there exists an $R>0$ such that the metric sphere $\partial B(p, \rho)$ is continuous in $\rho \in[0, R]$ with respect to the Hausdorff distance.

6.2. Space of components of metric circles. Assume that a number $R>0$ is small enough against a given point $p \in X$. Let $\mathcal{B}:=\bar{B}(p, R) / \sim$, where $\sim$ is the equivalence relation defined in the following: $x \sim y$ holds for two points $x, y \in$ $\bar{B}(p, R)$ if $d(p, x)=d(p, y)=: \rho$ and if $x$ and $y$ are contained in a common connected component of $\partial B(p, \rho)$. Recall that, by Lemma 6.2(1), any such component is either a point or a rectifiable circle. Denote by $[x]$ the equivalence class in $\mathcal{B}$ represented by a point $x \in \bar{B}(p, R)$. We put $\rho([x]):=\rho(x):=d(p, x)$ for any $x \in \bar{B}(p, R)$, and denote by $\mathcal{L}$ the set of liftable curves in $\mathcal{B}$, i.e., $\mathcal{L}:=\{\Pi \circ \tilde{\alpha} \mid$ $\tilde{\alpha}$ is a continuous curve in $\bar{B}(p, R)\}$, where $\Pi: \bar{B}(p, R) \rightarrow \mathcal{B}$ denotes the projection. Define the length $L(\alpha)$ of any curve $\alpha:[a, b] \rightarrow \mathcal{B}$ in $\mathcal{L}$ by

$$
L(\alpha):=\sup _{a=s_{0}<\cdots<s_{k}=b} \sum_{i=0}^{k-1}\left|\rho\left(\alpha\left(s_{i+1}\right)\right)-\rho\left(\alpha\left(s_{i}\right)\right)\right| .
$$

This induces an intrinsic (or length) metric $d$ on $X$ (cf. [11]), i.e., the distance $d([x],[y])$ between any two points $[x],[y] \in \mathcal{B}$ is defined by

$$
d([x],[y]):=\inf _{\alpha \in \mathcal{L}([x],[y])} L(\alpha),
$$

where $\mathcal{L}([x],[y])$ is the set of curves in $\mathcal{L}$ joining $[x]$ to $[y]$. It is easily verified that any curve $\alpha$ in $\mathcal{L}$ is continuous with respect to $d$ and its length induced from $d$ coincides with the original length $L(\alpha)$. It follows that the projection $\Pi: \bar{B}(p, R) \rightarrow \mathcal{B}$ is distance nonincreasing and hence $\mathcal{B}$ is compact.

In order to prove that $X$ is a pearl space, it is crucial to prove that $\mathcal{B}$ is isometric to a closed cone over a finite set (see Theorem 6.1).

It is easy to prove the following

Lemma 6.4. For any $x, y \in \bar{B}(p, R)$ we have

$$
\begin{gathered}
d([x],[y]) \geq|\rho([x])-\rho([y])|, \\
d([p],[x])=\rho([x])=L(\Pi(p x)) .
\end{gathered}
$$

In particular, $\Pi(p x)$ is a minimal segment in $\mathcal{B}$. 
Lemma 6.5. The closed metric ball $\bar{B}(p, R)$ is simply connected.

Proof. Suppose that $\bar{B}(p, R)$ is not simply connected. Then, there is a minimal loop $\gamma$ with base point $p$ in $\bar{B}(p, R)$ which is not homotopic to zero. Put $q:=$ $\gamma(L(\gamma) / 2)$. By Lemma 6.1(1), there exists a point $r \in X$ such that $\tilde{\angle} p q r \geq \pi-$ $\omega_{p}(R)$. Denote by $u, v \in \Sigma_{q}$ the directions at $q$ respectively corresponding to $\left.\gamma\right|_{[0, L(\gamma) / 2]}$ and $\left.\gamma\right|_{[L(\gamma / 2), L(\gamma)]}$. By $\angle(u, v)=\pi$ and Lemma 5.1, one has

$$
\min \left\{\angle\left(u, v_{q r}\right), \angle\left(v, v_{q r}\right)\right\} \leq \pi / 2+\omega_{p}(R) .
$$

Since Proposition 4.1 implies that the left-hand side of the above is $\geq \tilde{L} p q r-$ $\omega_{p}(R) \geq \pi-\omega_{p}(R)$, this is a contradiction. Therefore, $\bar{B}(p, R)$ is simply connected.

The following is a direct consequence of the lemma.

Corollary 6.2. Any liftable simple closed curve $\alpha \in \mathcal{L}([x],[x]),[x] \in \mathcal{B}$, is homotopic to zero.

Lemma 6.6. For any $\alpha \in \mathcal{L}$ there exists an injective curve $\alpha^{\prime} \in \mathcal{L}$ whose image is contained in that of $\alpha$.

Proof. It is obvious that there exists an injective continuous curve $\alpha^{\prime}$ whose image is contained in that of $\alpha$. The rest is to show that $\alpha^{\prime} \in \mathcal{L}$. Note that $\alpha^{\prime}$ is obtained by reparametrizing $\alpha$ and excluding at most countably many loops, say $\alpha_{i}, i=1,2, \ldots$. Let $\tilde{\alpha}$ be a lift of $\alpha$ into $\bar{B}(p, R)$, and $\tilde{\alpha}_{i}$ for each $i$ the lift of $\alpha_{i}$ which is a subarc of $\tilde{\alpha}$. Denote by $x_{i}$ and $y_{i}$ both the terminal points of $\tilde{\alpha}_{i}$. Notice then that $\left[x_{i}\right]=\left[y_{i}\right]$ holds for each $i$. To obtain a lift of $\alpha^{\prime}$ into $\bar{B}(p, R)$, we may replace each subarc $\tilde{\alpha}_{i}$ of $\tilde{\alpha}$ with some injective curve in $\Pi^{-1}\left(\left[x_{i}\right]\right)\left(=\Pi^{-1}\left(\left[y_{i}\right]\right)\right)$ joining $x_{i}$ and $y_{i}$.

Lemma 6.7. Let $[x],[y] \in \mathcal{B}$ and $\alpha, \beta \in \mathcal{L}([x],[y])$. Then, if $\alpha$ is injective, the image of $\alpha$ is contained in that of $\beta$.

Proof. Let $\alpha, \beta:[0,1] \rightarrow X \in \mathcal{L}([x],[y])$ and assume $\alpha$ is injective. Set $O:=$ $\{s \in[0,1] \mid \alpha(s) \notin \beta([0,1])\}$. It follows that $O$ is an open subset of the interval $(0,1)$. Pick up any $s \in O$ and fix it. By Lemma 6.2(1), the number of points $[z] \in \mathcal{B}$ such that $\rho([z])=\rho(\alpha(s))$ is finite, and hence there is a closed neighborhood $U$ of $\alpha(s)$ such that $\rho(U-\{\alpha(s)\}) \not \supset \rho(\alpha(s))$. The set $U-\{\alpha(s)\}$ is decomposed into the two disjoint subsets

$$
\begin{aligned}
& U_{+}:=\{[z] \in U \mid \rho([z])>\rho(\alpha(s))\}, \\
& U_{-}:=\{[z] \in U \mid \rho([z])<\rho(\alpha(s))\},
\end{aligned}
$$

which satisfy $\bar{U}_{+} \cap \bar{U}_{-}=\{\alpha(s)\}$. There is a $\delta>0$ such that $\alpha([s-\delta, s+\delta]) \subset U$. The injectivity of $\alpha$ yields that $\alpha([s-\delta, s) \cup(s, s+\delta]) \subset U_{+} \cup U_{-}$.

We now prove that $\rho \circ \alpha$ attains its local extremum at $s$. Suppose the contrary, so that, by reversing the parameter of $\alpha$ if necessary, one has $\alpha([s-\delta, s)) \subset U_{-}$and $\alpha((s, s+\delta]) \subset U_{+}$. The union of $\alpha$ and $\beta$ forms a simple closed curve, say $\gamma$, which is liftable into $X$ because there are two lifts $\tilde{\alpha}, \tilde{\beta}$ of $\alpha, \beta$ and one can find a curve joining $\tilde{\alpha}(0)$ to $\tilde{\beta}(0)$ (resp. $\tilde{\alpha}(1)$ to $\tilde{\beta}(1))$ contained in $\Pi^{-1}([x])$ (resp. $\left.\Pi^{-1}([y])\right)$. Therefore, by Corollary $6.2, \gamma$ is homotopic to zero. On the contrary, the intersection number of the curve $\gamma$ and the point $\alpha(s)$ is \pm 1 (depending on the orientation). This is a contradiction.

Thus, $\rho \circ \alpha$ attains its local extremum at any $s \in O$ and therefore $O=\emptyset$. 
The following proposition is a direct consequence of Lemmas 6.6 and 6.7.

Proposition 6.1. For any two points $[x],[y] \in \mathcal{B}$ we have:

(1) a minimal segment $[x][y]$ joining $[x]$ to $[y]$ uniquely exists and is an element of $\mathcal{L}$;

(2) the image of any curve in $\mathcal{L}([x],[y])$ contains the minimal segment $[x][y]$.

Proposition 6.2. Any minimal segment in $\mathcal{B}$ does not branch except at $[p]$.

Proof. Suppose there are $\left[x_{i}\right], i=1,2,3$, and $[y] \neq[p]$ in $\mathcal{B}$ such that $\left[x_{i}\right]$ and $[y]$ are all different and $\left[x_{1}\right]\left[x_{2}\right] \cap\left[x_{1}\right]\left[x_{3}\right]=\left[x_{1}\right][y]$. Proposition 6.1 proves $\left[x_{2}\right]\left[x_{3}\right]=$ $\left[x_{2}\right][y] \cup[y]\left[x_{3}\right]$. If $\Pi^{-1}([y])$ is homeomorphic to a circle, Lemma 6.2 shows that $[y]$ has a neighborhood homeomorphic to an arc, which is a contradiction. Therefore, $\Pi^{-1}([y])=\{y\}$, so that $x_{i} x_{j} \ni y$ for any $i \neq j$, which causes a contradiction by using the Triangle Comparison Theorem (1.5).

Letting $\partial \mathcal{B}:=\{[x] \in \mathcal{B} \mid \rho([x])=R\}$, we have:

Proposition 6.3. The minimal segment $[p][x]$ for any $[x] \in \mathcal{B}$ can be extended to $[p]\left[x^{\prime}\right]$ for some $\left[x^{\prime}\right] \in \partial \mathcal{B}$.

Proof. Set $R^{\prime}:=d(p, x)$ and $k:=\min \left\{i \in \mathbf{Z} \mid 2^{i} R^{\prime} \geq R\right\}$. Define $k+1$ points $x_{0}, \ldots, x_{k} \in X$ recursively by

$$
x_{i}:= \begin{cases}x & \text { if } i=0, \\ \Phi_{2^{i-1} R^{\prime}, 2^{i} R^{\prime}}\left(x_{i-1}\right) & \text { if } i<k, \\ \Phi_{2^{i-1} R^{\prime}, R}\left(x_{i-1}\right) & \text { if } i=k,\end{cases}
$$

where $\Phi$., is in Lemma 6.3. We will prove that $[p]\left[x_{i}\right] \subset[p]\left[x_{i+1}\right]$ for every $i$. Let $i$ be fixed. Lemma 1.5 implies that for any $y \in x_{i} x_{i+1}-\left\{x_{i}\right\}$ one has $\tilde{\angle} p x_{i} y \geq \tilde{L} p x_{i} x_{i+1}-$ $\omega_{p}(R) \geq \pi-\omega_{p}(R)$, so that $\rho\left(x_{i}\right)<\rho(y)$. This implies that $[p]\left[x_{i}\right] \cap \Pi\left(x_{i} x_{i+1}\right)=$ $\left\{\left[x_{i}\right]\right\}$. Since $\Pi\left(x_{i} x_{i+1}\right) \supset\left[x_{i}\right]\left[x_{i+1}\right]$, we have $[p]\left[x_{i}\right] \cap\left[x_{i}\right]\left[x_{i+1}\right]=\left\{\left[x_{i}\right]\right\}$, which together with Proposition 6.1(2) shows $[p]\left[x_{i}\right] \cup\left[x_{i}\right]\left[x_{i+1}\right]=[p]\left[x_{i+1}\right]$.

Therefore, $[p][x]=[p]\left[x_{0}\right] \subset[p]\left[x_{1}\right] \subset \cdots \subset[p]\left[x_{k}\right]$. Since $x_{k} \in \partial B(p, R),\left[x_{k}\right]$ is the desired $\left[x^{\prime}\right]$.

The compactness of $\mathcal{B}$ and Propositions 6.1, 6.2, and 6.3 together lead to the following:

Theorem 6.1. The space $\mathcal{B}$ is isometric to $\bar{B}(o, R ; \operatorname{cone} \partial \mathcal{B})$ and $\partial \mathcal{B}$ is a finite set.

Theorem 6.2. Any limit space $X \in \overline{\mathcal{M}(C, D)}$ is a pearl space.

Proof. Assume that a number $R>0$ is small enough against any fixed point $p \in X$. Theorem 6.1 implies that there are at most finitely many connected components of $B(p, R)-\{p\}$ and that the projection $L:=\Pi(S) \subset \mathcal{B}$ of each connected component $S$ of $B(p, R)-\{p\}$ is isometric to the open line segment of length $R$. Denote by $O$ the set of all $[x] \in L$ such that $\Pi^{-1}([x])$ is homeomorphic to a circle. Applying Lemma 6.2(2) to $\Gamma:=\Pi^{-1}([x])$ for every $[x] \in O$ yields that $O$ is an open subset of $L$ and the preimage $\Pi^{-1}\left(O^{\prime}\right)$ of any connected component $O^{\prime}$ of $O$ is homeomorphic to an open annulus. Since Corollary 6.1 implies the continuity of the map $L \ni[x] \mapsto$ $\Pi^{-1}([x]) \subset S$, the component $S$ is a string of pearls associated with the open subset $O \subset L$. This completes the proof. 
Remark 6.2. We observe that there is a natural correspondence between the connected components of $\Sigma_{p}$ and those of $B(p, R)-\{p\}$. In particular, $\operatorname{ind}(p)$ is equal to the number of components of $\Sigma_{p}$. Assume now that a component $S$ of $B(p, R)-\{p\}$ is corresponding to a circle component $\Gamma$ of $\Sigma_{p}$. Since $\left(\rho^{-1} \bar{S}, p\right)$ converges to $(K(\Gamma), o)$ as $\rho \rightarrow 0$, we have that $S \cap \partial B(p, \rho)$ for all sufficiently small $\rho \in(0, R]$ is homeomorphic to a circle, so that $S \cap B(p, \rho)$ is homeomorphic to an open annulus if $\rho>0$ is small enough.

6.3. Upper bound of the index and the fundamental group. As well as $c_{\mathrm{abs}}^{X}$, we can define the measure $c_{+}^{X}\left(\right.$ resp. $\left.c_{-}^{X}\right)$ over $X$ as a limit of $c_{+}^{M}$ (resp. $\left.c_{-}^{M}\right)$, where $M \in \mathcal{M}(C, D)$ tends to $X$. Define $c^{X}:=c_{+}^{X}-c_{-}^{X}$. These measures over $X$ are also not necessarily unique. It may be assumed that $c_{\mathrm{abs}}^{X}=c_{+}^{X}+c_{-}^{X}$.

Proposition 6.4. Let $X$ be a limit space of $\mathcal{M}(C, D)$. Then, the index at any point $p \in X$ satisfies

$$
\operatorname{ind}(p) \leq 2+\frac{1}{2 \pi} c_{-}^{X}(\{p\})
$$

and consequently,

$$
\sum_{p \in X} \max \{\operatorname{ind}(p)-2,0\} \leq \frac{1}{2 \pi} c_{-}^{X}(X) .
$$

Proof. There are an $M_{\delta} \in \mathcal{M}(C, D)$ and a measurable $\delta$-approximation $\varphi_{\delta}: M_{\delta} \rightarrow$ $X$ for any $\delta>0$ such that

$$
\lim _{\delta \rightarrow 0} \int_{M_{\delta}} f \circ \varphi_{\delta} d c_{ \pm}^{M_{\delta}}=\int_{X} f d c_{ \pm}^{X}
$$

for any continuous function $f$ on $X$. For a given point $p \in X$, one finds a point $p_{\delta} \in M_{\delta}$ in such a way that $d\left(\varphi_{\delta}\left(p_{\delta}\right), p\right)<\delta$. Let $\rho \in(0,1)$ be a number small enough against $p$ and $t \in\left[\rho^{2}, \rho\right]$ any number. We assume $\delta \leq \rho^{3}$. Then, since $\left(t^{-1} \bar{B}\left(p_{\delta}, t ; M_{\delta}\right), p_{\delta}\right)$ is close to $\left(\bar{B}\left(o, 1 ; K_{p}\right), o\right)$, the number of connected components of the metric sphere $\partial B\left(p_{\delta}, t\right)$ is at least ind $(p)$ (see Remark 6.2), so that in particular,

$$
\chi\left(B\left(p_{\delta}, t\right)\right) \leq 2-\operatorname{ind}(p) .
$$

Therefore, by (3.2),

$$
\begin{aligned}
0 & \leq \int_{0}^{\rho} \kappa\left(\partial B\left(p_{\delta}, t\right)\right) d t \\
& \leq \int_{0}^{\rho^{2}}(2 \pi+C) d t+\int_{\rho^{2}}^{\rho}\left\{2 \pi(2-\operatorname{ind}(p))+c_{-}^{M_{\delta}}\left(B\left(p_{\delta}, t\right)\right)\right\} d t,
\end{aligned}
$$

which implies

$$
\operatorname{ind}(p)<2+\left(1+\frac{C}{2 \pi}\right) \frac{1}{\rho^{-1}-1}+\frac{1}{2 \pi} c_{-}^{M_{\delta}}\left(B\left(p_{\delta}, \rho\right)\right) .
$$

Letting $\rho \rightarrow 0$ after $\delta \rightarrow 0$ completes the proof.

In order to prove (2) of the Topological Structure Theorem (1.2), we need some lemmas.

Lemma 6.8. Let $c:[0,1] \rightarrow X$ be a continuous curve in a metric space $X$. Then, for any $\epsilon>0$, there exists a sequence $0=s_{0}<s_{1}<\cdots<s_{k}=1$ such that $c\left(\left[s_{i}, s_{i+1}\right)\right) \subset B\left(c\left(s_{i}\right), \epsilon\right)$ for any $i=1, \ldots, k-1$. 
Proof. The lemma is implied by the uniform continuity of $c$.

Definition 6.1 ( $\epsilon$-locally 1-connectivity). A metric space $X$ is said to be $\epsilon$-locally 1-connected, $\epsilon>0$, if any closed curve with diameter $\leq \epsilon$ in $X$ is homotopic to zero.

Lemma 6.9. Let $X$ be an $\epsilon$-locally 1-connected intrinsic metric space, $\epsilon>0$, and let $\alpha, \beta:[0,1] \rightarrow X$ be two continuous curves such that $\alpha(0)=\beta(0)$ and $\alpha(1)=$ $\beta(1)$. Then, if $d(\alpha, \beta):=\sup _{s \in[0,1]} d(\alpha(s), \beta(s))<\epsilon / 3$, then $\alpha$ and $\beta$ are homotopic to each other fixing their terminal points.

Proof. By Lemma 6.8, there exists a sequence $0=s_{0}<\cdots<s_{k}=1$ such that $\operatorname{diam} \alpha\left(\left[s_{i}, s_{i+1}\right]\right), \operatorname{diam} \beta\left(\left[s_{i}, s_{i+1}\right]\right) \leq \epsilon / 6$. For every $i$ one finds a curve $\gamma_{i}$ joining $\alpha\left(s_{i}\right)$ and $\beta\left(s_{i}\right)$ and with length $L\left(\gamma_{i}\right) \leq \epsilon / 3$. Then, since the closed curve $\left.\left.\alpha\right|_{\left[s_{i}, s_{i+1}\right]} \cup \gamma_{i+1} \cup \beta\right|_{\left[s_{i}, s_{i+1}\right]} \cup \gamma_{i}$ for each $i$ has diameter $\leq \epsilon$, it is homotopic to zero. Therefore, $\alpha$ and $\beta$ are homotopic to each other.

Proposition 6.5. The fundamental group $\pi_{1}(X)$ of any limit space $X$ of $\mathcal{M}(C, D)$ is generated by at most $\left[2+c_{-}^{X}(X) / 2 \pi\right]$ elements.

Proof. Let $X$ be a limit space of $\mathcal{M}(C, D)$. It follows that there exists an $\epsilon>0$ such that $X$ is $\epsilon$-locally 1-connected. Find a number $\delta$ with $0<\delta \ll \epsilon$ and an $M \in \mathcal{M}(C, D)$ such that $d_{H}(M, X) \leq \delta$. Fix a base point $p_{0} \in M$ and set $x_{0}:=\varphi\left(p_{0}\right)$, where $\varphi: M \rightarrow X$ is a $2 \delta$-approximation. For a loop $\alpha:[0,1] \rightarrow M$ with base point $p_{0}$, we define a loop $\bar{\alpha}:[0,1] \rightarrow X$ with base point $x_{0}$ in the following. By Lemma 6.8, there exists a sequence $0=s_{0}<\cdots<s_{k}=1$ such that $\operatorname{diam} \alpha\left(\left[s_{i}, s_{i+1}\right]\right) \leq \delta$ for any $i$. Join $\varphi\left(\alpha\left(s_{i}\right)\right)$ to $\varphi\left(\alpha\left(s_{i+1}\right)\right)$ by a minimal segment $\bar{\alpha}_{i}$ for each $i$, and set $\bar{\alpha}:=\bigcup_{i} \bar{\alpha}_{i}$, which is a loop in $X$ with base point $x_{0}$. We parameterize $\bar{\alpha}$ so that $\bar{\alpha}\left(s_{i}\right)=\varphi\left(\alpha\left(s_{i}\right)\right)$ for any $i$.

We have the following:

Sublemma 6.1. If two loops $\alpha, \beta:[0,1] \rightarrow M$ with base point $p_{0}$ are homotopic to each other, then so are $\bar{\alpha}$ and $\bar{\beta}$.

Proof. Since $\alpha$ is homotopic to $\beta$, there exists a homotopy $H:[0,1] \times[0,1] \rightarrow M$ such that $H_{0}=\alpha$ and $H_{1}=\beta$, where $H_{t}:=H(\cdot, t):[0,1] \rightarrow M$ for $t \in[0,1]$. Applying Lemma 6.8 to $c:=t \mapsto H_{t}$ yields that there exists a sequence $0=$ $t_{0}<\cdots<t_{m}=1$ such that $d\left(H_{t_{i}}, H_{t_{i+1}}\right) \leq \delta$ for each $i$. We therefore have $d\left(\bar{H}_{t_{i}}, \bar{H}_{t_{i+1}}\right) \leq$ const $\delta<\epsilon / 3$, which together with Lemma 6.9 implies that $\bar{H}_{t_{i}}$ is homotopic to $\bar{H}_{t_{i+1}}$ for any $i$. This completes the proof of the sublemma.

By this sublemma, the correspondence $\pi_{1}(M) \ni[\alpha] \mapsto[\bar{\alpha}] \in \pi_{1}(X)$ defines a map $f: \pi_{1}(M) \rightarrow \pi_{1}(X)$. Notice that, although there are many choices of $\bar{\alpha}$ for a given $\alpha$, by Lemma 6.9 , the class $[\bar{\alpha}]$ is uniquely determined for the $\alpha$. It is easy to prove that $f$ is a homomorphism. We will show that $f$ is surjective. In fact, for any loop $\alpha$ in $X$ with base point $x_{0}$, one can define a loop $\bar{\alpha}$ in $M$ with base point $p_{0}$ as in the same manner as before. Since the definition of the loop $\overline{\bar{\alpha}}$ in $X$ implies $d(\overline{\bar{\alpha}}, \alpha) \leq$ const $\delta<\epsilon / 3$, and by Lemma 6.9 , the two loops $\alpha$ and $\overline{\bar{\alpha}}$ in $X$ are homotopic to each other and hence

$$
f([\bar{\alpha}])=[\overline{\bar{\alpha}}]=[\alpha] .
$$

Thus, $f$ is surjective. 
The homomorphism theorem yields that $\pi_{1}(X)$ is isomorphic to $\pi_{1}(M) / \operatorname{ker} f$. Moreover, since the Gauss-Bonnet theorem implies $-\chi(M) \leq c_{-}(M) / 2 \pi$, the fundamental group $\pi_{1}(M)$ is generated by at most $\left[2+c_{-}(M) / 2 \pi\right]$ elements. This completes the proof of Proposition 6.5.

Remark 6.3. In the proof of Proposition 6.5, the fundamental groups $\pi_{1}(M)$ and $\pi_{1}(X)$ are not necessarily isomorphic to each other. See Remark 4.1 for such an example. (1.2).

The following theorem is a stronger version of the Topological Structure Theorem

Theorem 6.3. Any limit space $X \in \overline{\mathcal{M}_{-}(C, D)}$ is a pearl space and satisfies the following (1) and (2):

(1) We have

$$
\sum_{p \in X} \max \{\operatorname{ind}(p)-2,0\} \leq \frac{C}{2 \pi} .
$$

(2) The fundamental group $\pi_{1}(X)$ of $X$ is generated by at most $[2+C / 2 \pi]$ elements.

Proof. If $X \in \overline{\mathcal{M}_{-}(C, D)}$, then $X \in \overline{\mathcal{M}(4 \pi+2 C, D)}$ (see Lemma 2.1) and hence, by Theorem $6.2, X$ is a pearl space. By remarking $c_{-}^{X}(X) \leq C,(1)$ and (2) follow from Propositions 6.4 and 6.5 respectively.

The same idea as in the proof of Proposition 6.5 leads to the following proposition, where we omit the proof.

Proposition 6.6. Let $X$ and $Y$ be two compact metric spaces which are $L G C(k, \rho)$ and $L G C(k-1, \rho)$ respectively. Then, there exists a $\delta>0$ depending only on $k$ and $\rho$ such that, if $d_{H}(X, Y)<\delta$, there is a surjective homomorphism from $\pi_{k}(Y)$ to $\pi_{k}(X)$.

Here, for the definition of $L G C(k, \rho)$ see [8].

Remark 6.4. We do not know whether any limit space of $\mathcal{M}(C, D)$ is $L G C(1, \rho)$ or not.

\section{Existence of the Limit Metric on PEARL SPACES}

In this section we provide:

Proof of Theorem 1.3. We first construct a metric on any given string of pearls. Let $\varphi: \mathbf{R} \rightarrow \mathbf{R}$ be a $C^{\infty}$-function such that

$$
\begin{array}{ll}
\varphi \equiv 0 & \text { on }(-\infty, 0] \cup[1,+\infty), \\
\varphi>0 & \text { on }(0,1) \\
\int_{0}^{1}\left|\varphi^{\prime \prime}(t)\right| d t=1
\end{array}
$$

For any $\epsilon>0$, set $\varphi_{\epsilon}(t):=\epsilon^{2} \varphi(t / \epsilon)$. Then, one has

$$
\int_{0}^{\epsilon}\left|\varphi_{\epsilon}^{\prime \prime}(t)\right| d t=\epsilon
$$


Let $O=\bigcup_{i \in I}\left(a_{i}, b_{i}\right) \subset(0,1)$ be any given open subset, where $\left(a_{i}, b_{i}\right)$ 's are all disjoint to each other. Define a function $f:(0,1) \rightarrow \mathbf{R}$ by

$$
f(t):= \begin{cases}0 & \text { for } t \in(0,1)-O, \\ \varphi_{b_{i}-a_{i}}\left(t-a_{i}\right) & \text { for } t \in\left(a_{i}, b_{i}\right) .\end{cases}
$$

The space

$$
S:=S(f):=\left\{(x, y, z) \in \mathbf{R}^{3} \mid y^{2}+z^{2}=f(x)^{2}, 0<x<1\right\}
$$

with its induced intrinsic metric is homeomorphic to the string of pearls associated with the open subset $O$.

We next see that $S$ can be approximated by a $C^{\infty}$-manifold homeomorphic to an annulus. For any $\epsilon>0$, put $I(\epsilon):=\left\{i \in I \mid b_{i}-a_{i} \geq \epsilon\right\}$ and $O(\epsilon):=\bigcup_{i \in I(\epsilon)}\left(a_{i}, b_{i}\right)$. Define a $C^{\infty}$-function $f_{\epsilon}:(0,1) \rightarrow \mathbf{R}$ by

$$
f_{\epsilon}(t):= \begin{cases}\epsilon & \text { for } t \in(0,1)-O(\epsilon), \\ \varphi_{b_{i}-a_{i}}\left(t-a_{i}\right)+\epsilon & \text { for } i \text { and } t \text { such that } b_{i}-a_{i} \leq \epsilon \text { and } t \in\left(a_{i}, b_{i}\right) .\end{cases}
$$

The $C^{\infty}$-surface of revolution $S_{\epsilon}:=S\left(f_{\epsilon}\right)$ is homeomorphic to an open annulus and converges to $S$ as $\epsilon \rightarrow 0$, while

$$
c_{\text {abs }}\left(S_{\epsilon}\right)=2 \pi \sum_{i \in I(\epsilon)}\left(b_{i}-a_{i}\right) \leq 2 \pi \quad \text { for any } \epsilon>0 .
$$

Now, let $X$ be a given compact pearl space, and $\left\{S_{i}\right\}$ the family of maximal strings of pearls embedded in $X$ as open subsets such that the boundary of each $S_{i}$ in $X$ consists of at most two points. The compactness of $X$ yields that $\#\left\{S_{i}\right\}<$ $\infty$. One observes that there are finitely many points $x_{1}, \ldots, x_{k} \in X$ such that $\hat{X}:=X-\bigcup_{i} \bar{S}_{i}-\left\{x_{1}, \ldots, x_{k}\right\}$ is a (not necessarily connected) finitely connected topological manifold without boundary, i.e., there is an embedding of $\hat{X}$ into a (not necessarily connected) compact 2-dimensional topological manifold $V$ such that $V-\hat{X}$ consists of finitely many points $e_{1}, \ldots, e_{m} \in V$, called the endpoints of $\hat{X}$. Find a $C^{\infty}$-Riemannian metric on $V$ and equip each string of pearls $S_{i}$ with the intrinsic metric defined above. This induces an intrinsic metric on $X$. (See Figure 5.)

We prove that $X \in \overline{\mathcal{M}(C, D)}$ in the following. In order to obtain a Riemannian manifold approximated to $X$, for $0<\epsilon \ll 1$, we replace the metric ball $B\left(p, \epsilon^{1 / 2}\right)$ centered at every $p \in T:=\bigcup_{i} \partial S_{i} \cup\left\{x_{1}, \ldots, x_{k}\right\}$ with the standard sphere of radius $\epsilon^{1 / 2}$ excluded $n$ disjoint disks with perimeter $2 \pi \epsilon$, where $n:=\operatorname{ind}(p)$. Notice here that $\epsilon$ is needed to be small enough against $n$. Replace every $S_{i}$ with $S_{i, \epsilon}$, where $S_{i, \epsilon}$ is the annulus $S_{\epsilon}$ defined above for $S=S_{i}$. Then, glue the circle boundaries of the replaced sphere excluded disks with all $\partial S_{i, \epsilon}$ and $\partial\left(\hat{X}-B\left(p, \epsilon^{1 / 2}\right)\right)$ in the manner as the original pieces did, so that we have a compact 2-dimensional topological manifold, say $X_{\epsilon}$, with piecewise $C^{\infty}$-Riemannian metric which is $\omega(\epsilon)$-close to $X$ with respect to the Gromov-Hausdorff distance. We deform the piecewise $C^{\infty}$ metric of $X_{\epsilon}$ slightly to obtain a $C^{\infty}$-Riemannian metric on $X_{\epsilon}$. It is possible to perform such a deformation satisfying that $d_{H}\left(X, X_{\epsilon}\right) \leq \omega(\epsilon)$ and

$$
C:=\sup _{0<\epsilon \ll 1} c_{\text {abs }}\left(X_{\epsilon}\right)<+\infty .
$$

Therefore, we have $\mathcal{M}(C, D) \ni X_{\epsilon} \rightarrow X$ as $\epsilon \rightarrow 0$ for $D:=2 \operatorname{diam} X$. 

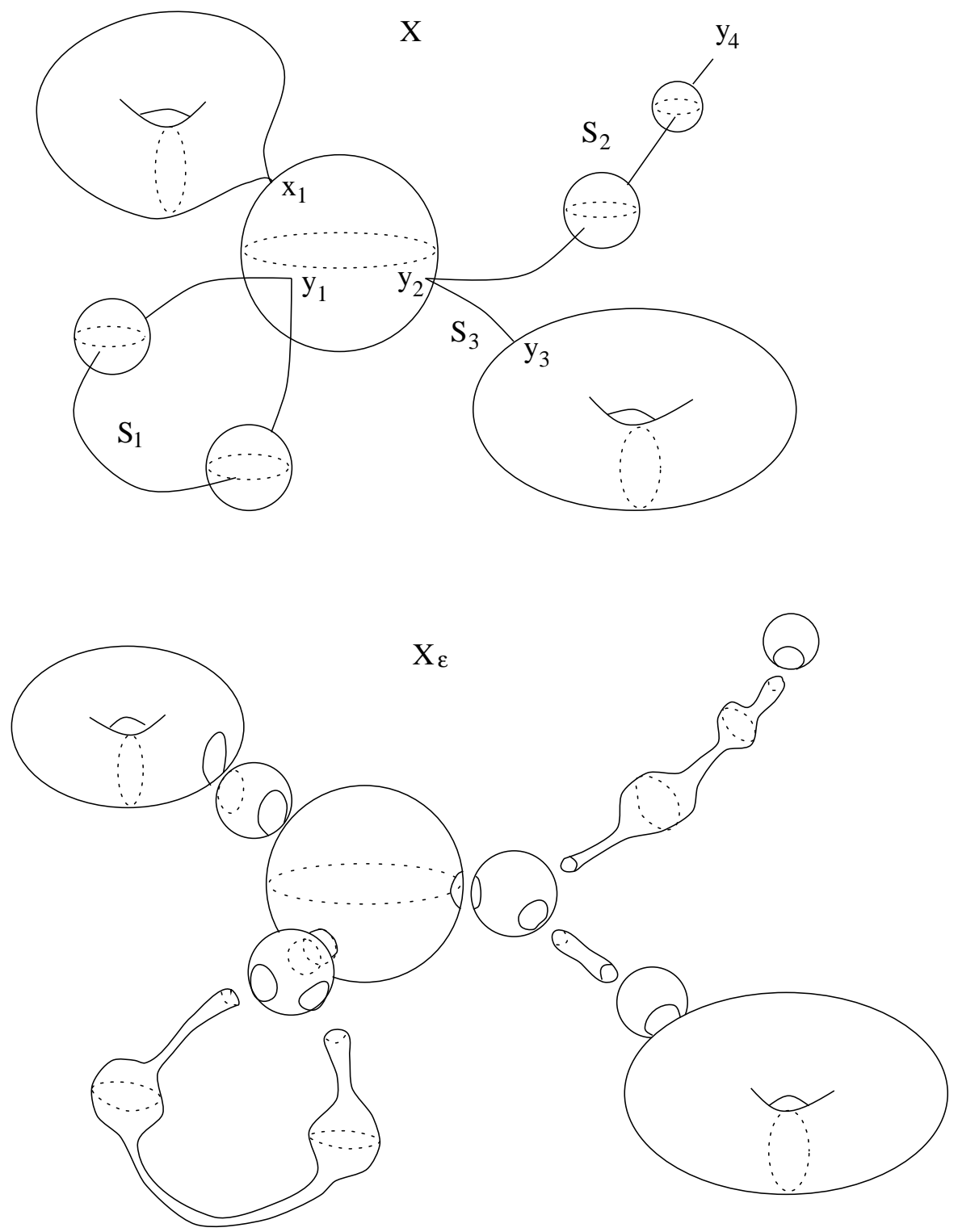

Figure 5

\section{Limit SPACES OF 2-MANIFOLDS WITH $L^{p}$-CURVATURE BOUND}

Throughout this section, let $p>1, C, D>0$ be given constants. Denote by $\mathcal{M}_{-}(p, C, D)$ the class of closed 2-dimensional Riemannian manifolds $M$ with diameter $\leq D$ and $L^{p}$-norm of negative curvature function $\int_{M} K_{-}{ }^{p} \leq C$. First we have the following:

Proposition 8.1. There exists a constant $C^{\prime}=\operatorname{const}_{p, C, D}$ such that

$$
\mathcal{M}(p, C, D) \subset \mathcal{M}_{-}(p, C, D) \subset \mathcal{M}_{-}\left(C^{\prime}, D\right) \subset \mathcal{M}\left(4 \pi+2 C^{\prime}, D\right) .
$$


Proof. The first implication is clear and the third has already been proved in Lemma 2.1. We will prove the second.

Let $M \in \mathcal{M}_{-}(p, C, D)$. Then, by Hölder's inequality, one has

$$
c_{-}(M) \leq\left(\int_{M} K_{-}{ }^{p}\right)^{1 / p}(\operatorname{vol} M)^{1 / q}
$$

where $q>1$ is defined by $1 / p+1 / q=1$. This together with Proposition 3.2(2) implies

$$
c_{-}(M) \leq \frac{C^{1 / p} D^{2 / q}}{2^{1 / q}}\left(2 \pi+c_{-}(M)\right)^{1 / q} .
$$

Therefore, there exists a constant $C^{\prime}=\operatorname{const}_{p, C, D}$ such that $c_{-}(M) \leq C^{\prime}$. This completes the proof.

Proposition 8.2. Any limit space $X$ of $\mathcal{M}_{-}(p, C, D)$ satisfies that $c_{-}^{X}(\{x\})=0$ for any $x \in X$.

Proof. Let $y \in M \in \mathcal{M}_{-}(p, C, D)$. Then, since $M \in \mathcal{M}_{-}\left(C^{\prime}, D\right), C^{\prime}=$ const $_{p, C, D}$, Proposition 3.2(2) implies

$$
\operatorname{vol} B(y, \rho) \leq \frac{1}{2}\left(2 \pi+C^{\prime}\right) \rho^{2} .
$$

Hence, by Hölder's inequality, for any $\rho>0$,

$$
c_{-}(B(y, \rho)) \leq\left(\int_{B(y, \rho)} K_{-}{ }^{p}\right)^{1 / p}(\operatorname{vol} B(y, \rho))^{1 / q} \leq \operatorname{const}_{p, C, D} \rho^{2 / q},
$$

where $1 / p+1 / q=1$.

For $x \in X \in \overline{\mathcal{M}_{-}(p, C, D)}$, there is a sequence of pointed manifolds $\left(M_{i}, y_{i}\right)$ with $M_{i} \in \mathcal{M}_{-}(p, C, D)$ such that $\left(M_{i}, y_{i}, c_{-}^{M_{i}}\right)$ tends to $\left(X, x, c_{-}^{X}\right)$ in the sense of the measured Gromov-Hausdorff convergence. Therefore, by (8.1) one has

$$
c_{-}^{X}(B(x, \rho)) \leq \operatorname{const}_{p, C, D} \rho^{2 / q},
$$

which completes the proof.

Theorem 8.1. Any limit space $X$ of $\mathcal{M}_{-}(p, C, D)$ is a compact pearl space and satisfies that $\operatorname{ind}(x) \leq 2$ for any $x \in X$.

Proof. Proposition 8.1 and Theorem 1.2 together imply that any limit space $X$ of $\mathcal{M}_{-}(p, C, D)$ is a compact pearl space. Combining Lemma 8.2 and Proposition 6.4 yields the estimate of the index.

Theorem 1.4 follows from Theorem 8.1 and the following:

Theorem 8.2. If a compact pearl space $X$ satisfies $\operatorname{ind}(x) \leq 2$ for any point $x \in X$, then $X$ has a metric such that $X \in \overline{\mathcal{M}(p, C, D)}$ for some $C, D>0$ depending on $p$ and $X$.

Proof. For a fixed number $p>1$, let $\varphi: \mathbf{R} \rightarrow \mathbf{R}$ be a $C^{\infty}$-function such that

$$
\begin{array}{cl}
\varphi \equiv 0 \quad \text { on }(-\infty, 0] \cup[1,+\infty) & \\
\varphi>0 \quad \text { on }(0,1) & \\
\int_{0}^{1} \frac{\left|\varphi^{\prime \prime}(t)\right|^{p}}{\varphi(t)^{p-1}} d t=1
\end{array}
$$


and set $\varphi_{\epsilon}(t):=\epsilon^{2 p}(\varphi(t / \epsilon)+\epsilon)$ for $t \in \mathbf{R}$ and $\epsilon>0$. Then, it follows that

$$
\int_{0}^{1} \frac{\left|\varphi_{\epsilon}^{\prime \prime}(t)\right|^{p}}{\varphi_{\epsilon}(t)^{p-1}} d t \leq \epsilon
$$

For a given open subset $O$ of $(0,1)$, we define the functions $f$ and $f_{\epsilon}$ in the same way as in the proof of Theorem 1.3. Define two more functions $f^{+-}:(-1 / 2,1+1 / 2) \rightarrow$ $\mathbf{R}$ and $f_{\epsilon}^{+-}:(-1 / 2,1+1 / 2) \rightarrow \mathbf{R}$ by

$$
\begin{aligned}
& f^{+-}(t):= \begin{cases}\varphi(t+1) & \text { for } t \in(-1 / 2,0], \\
f(t) & \text { for } t \in(0,1), \\
\varphi(t-1) & \text { for } t \in[1,1+1 / 2),\end{cases} \\
& f_{\epsilon}^{+-}(t):= \begin{cases}\varphi(t+1)+\epsilon & \text { for } t \in(-1 / 2,0], \\
f_{\epsilon}(t) & \text { for } t \in(0,1), \\
\varphi(t-1)+\epsilon & \text { for } t \in[1,1+1 / 2) .\end{cases}
\end{aligned}
$$

We set

$$
\begin{gathered}
f^{+}:=\left.f^{+-}\right|_{[0,1+1 / 2)}, \quad f^{-}:=\left.f^{+-}\right|_{(-1 / 2,1]}, \\
f_{\epsilon}^{+}:=\left.f_{\epsilon}^{+-}\right|_{[0,1+1 / 2)}, \quad f_{\epsilon}^{-}:=\left.f_{\epsilon}^{+-}\right|_{(-1 / 2,1]}, \\
S^{*}:=S\left(f^{*}\right), \quad S^{0}:=S /\{(0,0,0),(1,0,0)\}, \quad S_{\epsilon}^{+-}:=S\left(f_{\epsilon}^{+-}\right),
\end{gathered}
$$

where $*$ is any of '+', '-', '+-', or the empty character '. Let $S_{\epsilon}^{*}:=S\left(f_{\epsilon}^{*}\right) / \sim_{\epsilon}^{*}$ for $*=+,-$, ,, 0 , where $\sim_{\epsilon}^{*}$ is the equivalence relation on $S\left(f_{\epsilon}^{*}\right) \subset \mathbf{R}^{3}$ defined as follows: For any numbers $x, y, z \in \mathbf{R}$ such that $x=0,1$ and $y^{2}+z^{2}=\epsilon^{2}$, the relations

$$
\begin{array}{cc}
(0, y, z) \sim_{\epsilon}^{+}(0,-y,-z), & (1, y, z) \sim_{\epsilon}^{-}(1,-y,-z), \\
(x, y, z) \sim_{\epsilon}(x,-y,-z), & (0, y, z) \sim_{\epsilon}^{0}(1, y, z)
\end{array}
$$

are only defined to be true. It follows that $S_{\epsilon}^{ \pm}$is homeomorphic to the punctured projective plane (or the open Möbius strip), $S_{\epsilon}$ to the Klein bottle, and $S_{\epsilon}^{0}$ to the torus. For any $*=+,-,+-,{ }^{\prime}, 0$, the space $S_{\epsilon}^{*}$ is a $C^{\infty}$-Riemannian manifold and satisfies

$$
\int_{S_{\epsilon}^{*}}\left|K_{S_{\epsilon}^{*}}\right|^{p} \leq 4 \pi .
$$

Let $X$ be a compact pearl space such that ind $(x) \leq 2$ for any $x \in X$, and $\left\{S_{i}\right\}$ the family of strings of pearls in $X$ defined in the proof of Theorem 1.3. If $X$ is homeomorphic to $S^{0}$ associated with some open subset $O \subset(0,1)$, since $\mathcal{M}(p, C, D) \ni S_{\epsilon}^{0} \rightarrow S^{0}$ as $\epsilon \rightarrow 0$ for some $C, D>0$, this completes the proof. If not, there is a disjoint family of open neighborhoods $U_{i}$ of $\bar{S}_{i}$ such that each $U_{i}-\bar{S}_{i}$ consists of one or two punctured open disk domains and that $\hat{X}:=X-\bigcup_{i} U_{i}$ is a compact manifold with boundary. Each $U_{i}$ is homeomorphic to $S_{i}^{*}$, where $S_{i}^{*}$ is the string of pearls $S^{*}$ defined above for $S=S_{i}$ and $*$ is one of,,+-+- , or the empty character. Equip each $U_{i}$ with the intrinsic metric of $S_{i}^{*}$ and find a $C^{\infty}$-Riemannian metric on $\hat{X}$ such that the metric of $X$ is of $C^{\infty}$ around $\partial \hat{X}$. To obtain a $C^{\infty}$-Riemannian manifold $X_{\epsilon} \in \mathcal{M}(p, C, D)$ close to $X$, we replace each $U_{i}=S_{i}^{*}$ with $S_{i, \epsilon}^{*}$ and deform the metric on $\hat{X}$ slightly. It is easy to prove that 
$X_{\epsilon} \rightarrow X$ as $\epsilon \rightarrow 0$ and

$$
\sup _{0<\epsilon \ll 1} \int_{X_{\epsilon}}\left|K_{X_{\epsilon}}\right|^{p}<+\infty
$$

This completes the proof.

\section{REMARK ON METRIC SPACE OF BOUNDED INTEGRAL CURVATURE}

It seems natural to consider some concept of metric spaces with bounded total absolute curvature as a generalization of the limit spaces of $\mathcal{M}(C, D)$. One natural condition of such a metric space $X$ is that there is a Radon measure $c_{\text {abs }}^{X}$ over $X$ for which the conclusion of the Triangle Comparison Theorem (1.5) is satisfied. However, this condition does not imply the compactness of the spaces of directions of $X$, as is seen in the following examples:

Example 9.1. The subset of the complex plane $\mathbf{C}$

$$
X:=\left\{r e^{2^{-k} \pi \sqrt{-1}} \in \mathbf{C} \mid 0 \leq r \leq 2^{-k}, k=0,1, \ldots\right\}
$$

with its induced intrinsic metric is compact and satisfies the conclusion of the Triangle Comparison Theorem (1.5); however the space of direction $\Sigma_{o} X$ at the origin $o \in \mathbf{C}$ is an infinite and discrete set.

Another example is due to F. Ohtsuka ${ }^{1}$.

Example 9.2 (cf. Example 2.1 of [13]). Let $M$ be a complete Riemannian 2manifold and $F \subset M$ a compact subset. We define an intrinsic metric $d_{M / F}$ on the quotient space $M / F$ by

$$
d_{M / F}([p],[q]):=\min \{d(p, q), d(p, F)+d(q, F)\}
$$

for any $p, q \in M$, where $[p]$ is the class of $M / F$ represented by a point $p \in M$ and $d$ the distance function on $M$. Then, $\left(M / F, d_{M / F}\right)$ satisfies the Triangle Comparison Theorem (1.5). Moreover, the space of directions at the point $[F]$ is noncompact if and only if $F$ is an infinite subset.

We see [4], [5], and [21] for the study of metric spaces of bounded integral curvature.

\section{REFERENCES}

1. A. D. Alexandrov, A theorem on triangles in a metric space and some of its applications, Trudy Mat. Inst. Steklov. 38 (1951), 5-23. MR 14:198a

2. —_ Über eine Verallgemeinerung der Riemannschen Geometrie, Schriften Forschungsinst. Math. 1 (1957), 33-84.

3. A. D. Alexandrov, V. N. Berestovskii, and I. G. Nikolaev, Generalized Riemannian spaces, Russian Math. Surveys 41 (1986), no. 3, 1-54. MR 88e:53103

4. A. D. Alexandrov and V. A. Zalgaller, Intrinsic geometry of surfaces, Translations of Mathematical Monographs, vol. 15, Amer. Math. Soc., Providence, Rhode Island, 1967. MR 35:7267

5. Yu. D. Burago, Closure of the class of manifolds of bounded curvature, Proc. Steklov Inst. Math. 76 (1967), 175-183.

6. Yu. D. Burago, M. Gromov, and G. Perelman, A. D. Alexandrov spaces with curvature bounded below, Russian Math. Surveys 47 (1992), no. 2, 1-58. MR 93m:53035

7. H. Busemann, The geometry of geodesics, Academic Press, 1955. MR 17:779a

8. M. Cassorla, Approximating compact inner metric spaces by surfaces, Indiana Univ. Math. J. 41 (1992), 505-513. MR 93i:53042

\footnotetext{
${ }^{1}$ It is written in [21] that the example is due to Machigashira, which is however incorrect.
} 
9. X. Chen, Limit of metrics in Riemannian surfaces and the uniformization theorem, preprint, 1996.

10. K. Fukaya, Collapsing of riemannian manifolds and eigenvalues of Laplace operator, Invent. Math. 87 (1987), 517-547. MR 88d:58125

11. M. Gromov, J. Lafontaine, and P. Pansu, Structure métrique pour les variétés riemanniennes, Cedic/Fernand Nathan, Paris, 1981. MR 85e:53051

12. P. Hartman, Geodesic parallel coordinates in the large, Amer. J. Math. 86 (1964), 705-727. MR 30:3435

13. Y. Machigashira and F. Ohtsuka, Total excess on length surfaces, preprint, 1995.

14. G. Perelman, Elements of Morse theory on Alexandrov spaces, St. Petersburg Math. Jour. 5 (1994), no. 1, 207-214. MR 94h:53054

15. P. Petersen, S. Shteingold, and G. Wei, Comparison geometry with integral curvature bounds, Geom. Funct. Anal. 7 (1997), no. 6, 1011-1030. CMP 98:06

16. P. Petersen and G. Wei, Almost maximal volume and integral Ricci curvature bounds, preprint, 1996.

17. (1997), no. 6, 1031-1045. CMP 98:06

18. Yu. G. Reshetnyak (ed.), Geomtry IV, Encyclopaedia of Math. Sci., vol. 70, Springer-Verlag, 1993. MR 94i:53038

19. Two-Dimensional Manifolds of Bounded Curvature, 3-163, Vol. 70 of Encyclopaedia of Math. Sci. [18], 1993, pp. 3-163.

20. K. Shiohama and M. Tanaka, An isoperimetric problem for infinitely connected complete noncompact surfaces, Geometry of Manifolds (K. Shiohama, ed.), Perspectives in Math., vol. 8, Academic Press, 1989, pp. 317-343. MR 91b:53049

21. T. Shioya, The Gromov-Hausdorff limits of two-dimensional manifolds under integral curvature bound, Geometry and Topology: Proceedings of Workshops in Pure Mathematics, Vol. 16, Part III (1996) (Young Wook Kim, Sung-Eun Ko, Yongjin Song, Younggi Choi, eds.).

22. — Diameter and area estimates for $S^{2}$ and $P^{2}$ with nonnegatively curved metrics, Advanced Studies in Pure Math., vol. 22, Kinokuniya, Tokyo, 1993, pp. 309-319. MR 95h:53055

23. M. Trojanov, Un principe de concentration-compacite pour les suites de surfaces riemaniennes, Ann. Inst. Henri Poincaré 8 (1991), no. 5, 1-23. MR 92m:53077

24. D. Yang, Convergence of Riemannian manifolds with integral bounds on curvature I, Ann. Sci. École Norm. Sup. (4) 25 (1992), 77-105. MR 93a:53037

Graduate School of Mathematics, Kyushu University, Fukuoka 812-8581, Japan

E-mail address: shioya@math.kyushu-u.ac.jp 